\title{
IMPACT OF THE VARIATIONS OF THE MIXING LENGTH IN A FIRST ORDER TURBULENT CLOSURE SYSTEM
}

\author{
FrANÇOISE BROSSIER $^{1}$ AND Roger LEWANDOWSKI ${ }^{2}$
}

\begin{abstract}
This paper is devoted to the study of a turbulent circulation model. Equations are derived from the "Navier-Stokes turbulent kinetic energy" system. Some simplifications are performed but attention is focused on non linearities linked to turbulent eddy viscosity $\nu_{t}$. The mixing length $\ell$ acts as a parameter which controls the turbulent part in $\nu_{t}$. The main theoretical results that we have obtained concern the uniqueness of the solution for bounded eddy viscosities and small values of $\ell$ and its asymptotic decreasing as $\ell \rightarrow \infty$ in more general cases. Numerical experiments illustrate but also allow to extend these theoretical results: uniqueness is proved only for $\ell$ small enough while regular solutions are numerically obtained for any values of $\ell$. A convergence theorem is proved for turbulent kinetic energy: $k_{\ell} \rightarrow 0$ as $\ell \rightarrow \infty$, but for velocity $u_{\ell}$ we obtain only weaker results. Numerical results allow to conjecture that $k_{\ell} \rightarrow 0, \nu_{t} \rightarrow \infty$ and $u_{\ell} \rightarrow 0$ as $\ell \rightarrow \infty$. So we can conjecture that this classical turbulent model obtained with one degree of closure regularizes the solution.
\end{abstract}

Mathematics Subject Classification. 35Q30, 76M10, 76DXX, 76FXX, 46TXX, 65NXX.

Received: October 3, 2001.

\section{INTRODUCTION}

It is necessary to define a correct representation for the generation of turbulent eddies in order to obtain physically significant modelizations of geophysical flows. We study in this paper a very simplified example of turbulent model but where the main mathematical difficulties arising in one degree of closure turbulent systems are still present in the simplified equations.

In Section 2 we recall how is obtained the system of equations representing a turbulent flow. Velocity $u$ appearing in the model corresponds to a statistical mean of the turbulent velocity. Turbulent eddy viscosity $\nu_{t}$ is defined as $\nu_{t}=C+\ell k^{1 / 2}$ where $k$ is the turbulent kinetic energy, $C$ a given constant and $\ell$ can be interpreted as the mixing length. The closure assumptions allow to obtain the equation satisfied by $k$. The one degree of closure turbulent model coupled with primitive equations of geophysical flows gives rise to a set of Navier-Stokes type equations called "Naviers-Stokes Turbulent Kinetic Energy" (NSTKE) system (see below). The NSTKE system is time-dependent, 3-dimensional and strongly non linear. Very few theoretical results can be obtained for this set of equations (see for example $[3,5,8,9]$ ). When the existence of a solution can be proved, the uniqueness is not known and there are no results either on the regularity of solutions or on their asymptotic behaviour. This lack of theoretical results sets serious problems for interpreting the results of any numerical

\footnotetext{
Keywords and phrases. Turbulence modelling, energy methods, mixing length, finite-elements approximations.

1 IRMAR, INSA, Campus de Beaulieu, 35043 Rennes Cedex, France.

2 IRMAR, Université de Rennes 1, Campus de Beaulieu, 35042 Rennes Cedex, France.
} 
simulation and for validation of $(k-\varepsilon)$ models. Validity of numerical results has to be verified. For example if a numerical model produces some instabilities, it is essential to distinguish physical instabilities due to turbulence from numerical instabilities due to the discretization scheme. The existence of instabilities can also be linked to the modelization of turbulence. The closure model can either smooth the solution or generate instabilities. We underline the importance of a previous analysis of the behaviour of the model before interpreting the results of such numerical simulations of turbulent flows. This explains the process followed in this paper. Because of the complexity of the NSTKE system it is actually impossible to analyse a realistic modelization of turbulent geophysical flows. So we are going to work with a very simplified turbulent model as a mathematical laboratory. We seek for $(u, k)$ solution of the following set of equations

$$
\left\{\begin{array}{l}
-\nabla \cdot\left(\nu_{t} \nabla u\right)=f, \\
-\nabla \cdot\left(\nu_{t} \nabla k\right)=\nu_{t}|\nabla u|^{2}-\frac{1}{\ell} k^{3 / 2},
\end{array}\right.
$$

in $\Omega$ which is a bounded domain in $\mathbb{R}^{N}$ (N=2 or 3 ). The couple $(u, k)$ has to satisfy Dirichlet or Neumann boundary conditions on $\partial \Omega$. Most of the theoretical results remain valid for more realistic models as those considered in numerical Section 4. We mention that additional results are known about regularity of solutions in particular two-dimensional cases (see [3]).

System $\left(\mathcal{S}_{1}\right)$ is steady-state, which is justified if we want to obtain the flow driven by a stationary forcing. It is then physically reasonable to assume that the mean statistical flow is stationary. Convection terms $(u . \nabla) u$ and $(u . \nabla) k$ have been neglected but non-linearities linked to turbulent viscosity are still present in $\left(\mathcal{S}_{1}\right)$. Of course this model cannot describe a complex realistic flow but it can allow to understand the mathematical and numerical behaviours of the basic terms appearing in a one degree of closure turbulent model. From a mathematical view point main difficulties come from the following non-linearities: $\nabla \cdot\left(k^{1 / 2} \nabla(u, k)\right)$ corresponding to turbulent dissipation, $k^{1 / 2}|\nabla u|^{2}$ which represents energy transfers from large to small scales and $\frac{1}{\ell} k^{3 / 2}$ which represents small scales dissipation.

One of the questions we want to answer concerns the relationship between the turbulent viscosity coefficient and the nature of the solution. The value of the mixing length $\ell$ is a parameter which controls the turbulent part in $\nu_{t}$. Does increasing values of $\ell$ give rise to bifurcations between different branches of solutions or does it give a unique regular solution corresponding to the mean flow?

Section 3 is devoted to mathematical results, on one hand for small values of $\ell$, on the other hand for large values of $\ell$. The main theoretical result of the paper is the uniqueness result proved in Theorem 3.2 for $\ell$ small enough. In order to prove this result, we have to assume that $\nu_{t}=1+\ell a(k)$, where $a(k)$ is a $C^{1}$ non negative bounded function, and to neglect the dissipation term $\varepsilon=-\frac{1}{\ell} k^{3 / 2}$. The general case is still an open problem. Notice that the system of equations deduced from $\left(\mathcal{S}_{1}\right)$ by neglecting the $\varepsilon$ term is used in electromagnetism. In this case $u$ represents the temperature and $k$ the magnetic field (see $[3,5]$ and references inside). Unfortunately the method used for uniqueness doesn't work when the $\varepsilon$ term is present or when $a(k)$ is not bounded as in the case $a(k)=k^{1 / 2}$ and then the problem remains open. Afterwards we study the asymptotic behaviour of $\left(u_{\ell}, k_{\ell}\right)$, solution of $\left(S_{1}\right)$ when $\ell \rightarrow \infty$. Theorem 3.3 proves that the turbulent kinetic energy $k_{\ell}$ converges towards 0 as $\ell \rightarrow \infty$. At the present time convergence results for the velocity $u_{\ell}$ are weaker. In some sense, the result we can prove in general cases is some kind of equivalence between the two following convergences: $u_{\ell} \rightarrow 0$ and $\nu_{t}^{\ell} \rightarrow \infty$ as $\ell \rightarrow \infty$, where $\nu_{t}^{\ell}$ denotes the corresponding eddy viscosity. Moreover we can prove that $u_{\ell} \rightarrow 0$ as $\ell \rightarrow \infty$ if the eddy viscosity $\nu_{t}^{\ell}$ is given by $1+\ell b(k)$ with $b(0) \neq 0$. Physical observations as well as numerical results allow to conjecture that these two convergence results $u_{\ell} \rightarrow 0$ and $\nu_{t}^{\ell} \rightarrow \infty$ as $\ell \rightarrow \infty$ are true even in the case $\nu_{t}^{\ell}=1+\ell k^{1 / 2}$. It has to be noted that for large values of $\ell$ the term $\varepsilon=-\frac{1}{\ell} k^{3 / 2}$ appearing in $\mathcal{S}_{1}$ is negligible.

Section 4 is devoted to numerical results. Two different numerical experiments have been carried out. The first one solves system $\left(\mathcal{S}_{1}\right)$ in a three-dimensional domain $\Omega$. The second one solves in a two-dimensional domain a more realistic problem since including vectorial velocity, pressure gradient and continuity equation. In the 
two cases we have studied the behaviour of the kinetic energy $k_{\ell}$, of the turbulent viscosity $\nu_{t}^{\ell}=1+\ell k^{1 / 2}$ and of the velocity $u_{\ell}$ when the mixing length $\ell$ varies between 0 and 100 . For any values of $\ell$ we obtain stable numerical solutions which backs up the hypothesis of uniqueness of the solution even if $\ell$ is large. Turbulent kinetic energy $k_{\ell}$ is maximum for values of $\ell$ between 0.05 and 0.1 ; for $\ell$ greater than $0.1, k_{\ell}$ decreases and tends to 0 as $\ell$ increases. Turbulent viscosity $\nu_{t}^{\ell}$ is an increasing function of $\ell$, velocity $u_{\ell}$ a decreasing function. Numerical results confirm the conjecture about convergences: $u_{\ell} \rightarrow 0$ and $\nu_{t}^{\ell} \rightarrow \infty$ as $\ell \rightarrow \infty$.

\section{SetTing OF THE MODEL}

We first recall the classical Reynolds problem.

Let $(\mathcal{W}, \mathcal{A}, \mathcal{P})$ be a probabilistic space, $\Omega \subset \mathbb{R}^{3}$ being a volume of control. Given a flow

$$
u=u(w, t, x), \quad w \in \mathcal{W}, t \in \mathbb{R}_{+}, x \in \Omega,
$$

one define the mean value of $u$ at time $t$ and position $x$ by

$$
\underline{u}(t, x)=E(u(w, t, x))=\int_{\mathcal{P}} u(w, t, x) \mathrm{d} \mathcal{P}(w) .
$$

Thus, one can write

$$
u=\underline{u}+u^{\prime}
$$

where

$$
E\left(u^{\prime}\right)=\underline{u}^{\prime}=0 .
$$

Notice that if $u \in L^{1}\left(\mathcal{W} \times \mathbb{R}_{+} \times \Omega\right)$ one has in a distribution sense

$$
\frac{\partial \underline{u}}{\partial x_{i}}=E\left(\frac{\partial u}{\partial x_{i}}\right), \quad \frac{\partial \underline{u}}{\partial t}=E\left(\frac{\partial u}{\partial t}\right) .
$$

We now assume that $u$ verifies the incompressible Navier-Stokes equations

$$
\left\{\begin{array}{l}
\partial_{t} u+(u . \nabla) u=-\frac{\nabla p}{\rho}+\nu \Delta u \\
\nabla \cdot u=0
\end{array}\right.
$$

where $\rho$ is the density of the flow, assumed to be constant, $p$ the pressure (including the body forces deriving from a potential) and $\nu$ is the kinematic viscosity coefficient. For the sake of the simplicity and without loss of generality, we shall assume that $u$ satisfies a no slip condition on the boundary, that is to say

$$
u=0 \quad \text { on } \partial \Omega .
$$

We write $p=\underline{p}+p^{\prime}$ and average equations (2) by taking the esperance $E$. Thanks to (1), this leads to

$$
\left\{\begin{array}{l}
\partial_{t} \underline{u}+(\underline{u} . \nabla) \underline{u}=-\frac{\nabla \underline{p}}{\rho}+\nu \Delta \underline{u}-\nabla \cdot\left(\underline{u^{\prime} \otimes u^{\prime}}\right), \\
\nabla \cdot \underline{u}=0 .
\end{array}\right.
$$


The tensor $-\underline{u^{\prime} \otimes u^{\prime}}$ is called the Reynolds stress. The strong dissipative character of the turbulence in regions of high velocity gradients suggests that the Reynolds stress is proportional to the mean rate strain tensor, which means (see [13])

$$
-\underline{u^{\prime} \otimes u^{\prime}}=\nu_{t}\left(\nabla \underline{u}+\nabla \underline{u}^{T}\right),
$$

where $\nu_{t}$ is the eddy viscosity coefficient.

Relation (5) is also called the Reynolds hypothesis. Thus system (4) becomes

$$
\left\{\begin{array}{l}
\partial_{t} \underline{u}+(\underline{u} . \nabla) \underline{u}=-\frac{\nabla \underline{p}}{\rho}+\nabla \cdot\left(\nu_{t}\left(\nabla \underline{u}+\nabla \underline{u}^{T}\right)\right)+\nu \Delta \underline{u}, \\
\nabla \cdot \underline{u}=0 .
\end{array}\right.
$$

In order to close system (6), we now have to determine the eddy viscosity $\nu_{t}$. The classical first order closure hypothesis suggests that $\nu_{t}$ is only function of the turbulent kinetic energy $k$ (TKE) defined by

$$
k=\frac{1}{2}{\underline{u^{\prime}}}^{2}=E\left(\frac{1}{2}\left|u^{\prime}\right|^{2}\right) .
$$

By a dimension analysis, it is easy to see that the only formula for $\nu_{t}$ is given by

$$
\nu_{t}=\ell k^{1 / 2},
$$

where $\ell$ has the dimension of a length. Following Kolmogorov, we can substitute to the $(k, \varepsilon)$ description of the turbulence a description using $k$ and the mixing length. Thus $\ell$ appearing in (7) can only be interpreted as the mixing length, related to $k$ and $\varepsilon$ by the formula aforementioned

$$
\varepsilon=\frac{1}{\ell} k^{3 / 2}
$$

In the following $\ell$ will be used as a free parameter measuring the intensity of turbulence.

In order to obtain the equation verified by the turbulent kinetic energy $k$, we have to assume that the following hypothesis are satisfied.

(H1) Reynolds hypothesis (5),

(H2) ergodicity of the field $u$ in order to replace esperances by space means when necessary, that is for instance, for a ball $B$,

$$
\begin{aligned}
E\left(u^{\prime} \Delta u^{\prime}\right) & \approx \frac{1}{|B|} \int_{B} u^{\prime} \Delta u^{\prime}=\frac{1}{|B|} \int_{\partial B} u^{\prime} \nabla u^{\prime} \cdot n-\frac{1}{|B|} \int_{B}\left|\nabla u^{\prime}\right|^{2}=\int_{\partial B} u^{\prime} \nabla u^{\prime} \cdot n-\frac{\varepsilon}{\nu}, \\
E\left(\nabla \cdot\left(p^{\prime} u^{\prime}\right)\right) & \approx \frac{1}{|B|} \int_{\partial B} p^{\prime} u^{\prime} \cdot n,
\end{aligned}
$$

(H3) isotropy of $u^{\prime}$ in order to neglect all odd boundary integrals after integration by parts, which means

$$
\int_{\partial B} u^{\prime} \nabla u^{\prime} \cdot n \approx \int_{\partial B} p^{\prime} u^{\prime} \cdot n \approx 0
$$

(H4) convection by random fields gives rise to diffusion after averaging, that is to say

$$
E\left(\partial_{t} e+\left(\underline{u}+u^{\prime}\right) \nabla e\right) \approx \partial_{t} \underline{e}+\underline{u} . \nabla \underline{e}-\nabla \cdot\left(\tilde{\nu}_{t} \nabla \underline{e}\right), \text { for any scalar quantity } e .
$$

The new eddy viscosity $\tilde{\nu}_{t}$ is proportional to $\nu_{t}$. 
The equation satisfied by $k$ under hypothesis (H1) to (H4) is (see [13] for instance)

$$
\partial_{t} k+\underline{u} . \nabla k-\nabla \cdot\left(\tilde{\nu}_{t} \nabla k\right)=\nu_{t}\left|\nabla \underline{u}+\nabla \underline{u}^{T}\right|^{2}-\varepsilon,
$$

where $\varepsilon=\nu E\left(\left|\nabla u^{\prime}+\nabla u^{\prime T}\right|^{2}\right)=\frac{1}{\ell} k^{3 / 2}$, as already mentioned, represents the dissipation due to the small scales.

In summary the system deduced from these arguments and known as Navier-Stokes turbulent kinetic energy system (NSTKE) is

$$
\text { (NSTKE) }\left\{\begin{array}{l}
\partial_{t} \underline{u}+(\underline{u} . \nabla) \underline{u}=-\frac{\nabla \underline{p}}{\rho}+\nabla \cdot\left(\nu_{t}\left(\nabla \underline{u}+\nabla \underline{u}^{T}\right)\right)+\nu \Delta \underline{u} \\
\partial_{t} k+(\underline{u} . \nabla) k=\nabla \cdot\left(\tilde{\nu}_{t} \nabla k\right)+\nu_{t}\left|\nabla \underline{u}+\nabla \underline{u}^{T}\right|^{2}-C_{3} \frac{k^{3 / 2}}{\ell}, \\
\nabla \cdot \underline{u}=0 .
\end{array}\right.
$$

Nota bene: notation "-" used for mean values of instant velocity u will now be omitted.

Very few mathematical results are known on system (NSTKE), even in the case of boundary conditions as simple as homogeneous Dirichlet boundary conditions. When $\nu_{t}$ is assumed to be smooth and bounded (which is not the case in the reality of formula (7)), existence of a weak solution is obtained in the two dimensional case, in the steady-state case or when the convective terms are neglected (see [9]). It was also conjectured in [8] and [9] that in some 3D cases, a positive measure can appear in the TKE equation by passing to the limit. This conjecture is confirmed by a recent work of Duchon-Robert [4] and is actually investigated in [10].

The system we are going to study in this paper is a simplified version of the (NSTKE) system. We are looking for a mean flow driven by stationary forces. So we neglect time-dependence and consider a steady-state system. Convective terms $(u . \nabla) u$ and $(u . \nabla) k$ are also neglected because we want to focus on non linearities linked to the turbulent viscosity closure model. In Section 3 we study some mathematical properties for a system including the turbulent dissipation terms $\nabla \cdot\left(\nu_{t} \nabla u\right)$, and $\nabla \cdot\left(\nu_{t} \nabla k\right)$, and the term $\nu_{t}|\nabla u|^{2}$ which represents energy transfers from large to small scales. We still denote by $\nu_{t}$ the quantity $\nu+\nu_{t}$ and assume that it is also equal to $\tilde{\nu}_{t}$. Without losing generality, we put $\nu_{t}=1+\ell k^{1 / 2}$.

Nevertheless, we are constrain in Section 3.1 below to truncate the eddy viscosity in reason of theoretical obstructions. Thus, it will be replaced by $\nu_{t}=1+\ell a(k)$ where $a(k)$ is a $C^{1}$ non negative bounded function.

\section{Theoretical Results}

We consider the following system

$$
\begin{cases}-\nabla \cdot\left(\nu_{t}(k) \nabla u\right)=f & \text { in } \Omega \\ -\nabla \cdot\left(\nu_{t}(k) \nabla k\right)=\nu_{t}(k)|\nabla u|^{2} & \text { in } \Omega, \\ k=u=0 & \text { on } \partial \Omega \\ k \geqslant 0 & \text { in } \Omega,\end{cases}
$$

where $\nu_{t}(k)=1+\ell k^{1 / 2}, \Omega \subseteq \mathbb{R}^{N}, N=2,3$.

We shall assume that $f \in L^{2}(\Omega)$ and $N=3$, the case $N=2$ being simpler.

Definition 3.1. We shall say that $(u, k)$ is an energy solution to $\left(\mathcal{S}_{2}\right)$ if and only if

$$
\begin{gathered}
u \in H_{0}^{1}(\Omega), \quad k \in \underset{p<N^{\prime}}{\cap} W_{0}^{1, p}(\Omega), \quad \nu_{t}|\nabla u|^{2} \in L^{1}(\Omega), \\
\forall v \in H_{0}^{1}(\Omega) \text { such that } \nu_{t}(k)|\nabla v|^{2} \in L^{1}(\Omega), \quad \int \nu_{t}(k) \nabla u . \nabla v=\int f . v,
\end{gathered}
$$




$$
\forall \phi \in C_{c}^{\infty}(\Omega), \quad \int \nu_{t}(k) \nabla k . \nabla \phi=\int \nu_{t}(k)|\nabla u|^{2} \phi .
$$

Theorem $3.1([6])$. System $\left(\mathcal{S}_{2}\right)$ admits at least one energy solution $\left(u_{\ell}, k_{\ell}\right)$. Moreover, there exists $G=$ $G\left(\|f\|_{L^{2}}, \Omega\right)$ such that

$$
\int \nu_{t}\left(k_{\ell}\right)\left|\nabla u_{\ell}\right|^{2} \leqslant G
$$

and there exists $D_{p}=D_{p}\left(p,\|f\|_{L^{2}}, \Omega\right)$ with $D_{p} \underset{p \rightarrow N^{\prime}}{\rightarrow}+\infty$ such that

$$
\left\|k_{\ell}\right\|_{W_{0}^{1, p}(\Omega)} \leqslant D_{p} .
$$

It has to be noted that estimates (12) and (13) do not involve $\ell$.

Remark 3.1. Assume now that $\nu_{t}$ is a bounded function of $k$. Then the notion of energy solution coincides with the classical notion of weak solution. The equation for $u$ then holds in the weak sense in the meaning of Leray-Lions and the equation for $k$ holds in the distribution sense.

For the proof of this result, see [6] combined with Proposition 5.2.1, p. 185 in [8].

In what follows, we shall first study the case of small values of the mixing length $\ell$ where the eddy viscosity is bounded and smooth, then we shall consider the problem of asymptotic behaviour of the solution for large values of $\ell$.

\subsection{Case of small values of $\ell$ : uniqueness result}

Throughout this subsection, we assume that

$$
\nu_{t}=\nu_{t}(k)=1+\ell a(k),
$$

where $a(k)$ is a $C^{1}$ non negative bounded function. We shall prove that system $\left(S_{2}\right)$ has a unique solution for $\ell$ small enough. Before doing that, we start with the study of the sequences of solutions when $\ell$ goes to zero. First let consider the system

$$
\begin{cases}-\Delta u=f & \text { in } \Omega, \\ -\Delta k=|\nabla u|^{2} & \text { in } \Omega, \\ u=k=0 & \text { on } \partial \Omega \\ k \geqslant 0 & \text { in } \Omega,\end{cases}
$$

corresponding to the case $\ell=0$. Because $f \in L^{2}(\Omega)$ and $\Omega$ is a smooth domain, it is obvious that $\left(S_{0}\right)$ has a unique solution

$$
\left(u_{0}, k_{0}\right) \in\left[H_{0}^{1}(\Omega) \cap H^{2}(\Omega)\right]^{2} .
$$

Let $p_{0}<N^{\prime}$ fixed and close enough to $N^{\prime}$. Put

$$
\mathcal{X}=H_{0}^{1}(\Omega) \times W_{0}^{1, p_{0}}(\Omega) .
$$


We denote by $A_{\ell}$ the set of all solutions of system $\left(S_{2}\right)$ corresponding to the parameter $\ell$. According to Theorem 3.1 and Remark 3.1, $A_{\ell} \neq \emptyset$ and

$$
A_{\ell} \subseteq H_{0}^{1}(\Omega) \times\left(\underset{p<N^{\prime}}{\cap} W_{0}^{1, p}(\Omega)\right)
$$

In particular

$$
A_{\ell} \subseteq \mathcal{X}
$$

Let $\left(\ell_{n}\right)_{n \in \mathbb{N}}$ be a sequence of non negative real numbers which converges towards zero, let $x_{n}=\left(u_{n}, k_{n}\right) \in A_{\ell_{n}}$ be an arbitrary chosen solution of system $\left(S_{2}\right)$.

Lemma 3.1. The sequence $\left(x_{n}\right)_{n \in \mathbb{N}}=\left(u_{n}, k_{n}\right)_{n \in \mathbb{N}}$ converges towards $x_{0}=\left(u_{0}, k_{0}\right)$ solution of problem $\left(\mathcal{S}_{0}\right)$, strongly in the space $\mathcal{X}$.

Corollary 3.1. Given any neighbourhood $V$ of $x_{0}$ in $\mathcal{X}$, there exists $\ell_{0}>0$ such that $\forall \ell \in\left[0, \ell_{0}\left[, A_{\ell} \subseteq V\right.\right.$.

Proof. Because of estimates (12) and (13) and thanks to the positiveness of the function $a(k)$, the sequence $\left(x_{n}\right)_{n \in \mathbb{N}}$ is bounded in

$$
H_{0}^{1}(\Omega) \times \underset{p<N^{\prime}}{\cap} W_{0}^{1, p}(\Omega) .
$$

Then, from the sequence $\left(u_{n}\right)_{n \in \mathbb{N}}$, one can extract a subsequence (still denoted $\left.\left(u_{n}\right)_{n \in \mathbb{N}}\right)$ converging to a function $\underline{u}_{0} \in H_{0}^{1}(\Omega)$ as $n \rightarrow+\infty$, weakly in $H_{0}^{1}(\Omega)$, strongly in $L^{q}(\Omega), \forall q<6$ if $N=3, \forall q<+\infty$ if $N=2$, and almost everywhere in $\Omega$. Moreover, arguing as in [8], Corollary 5.3.1, page 190, from the sequence $\left(k_{n}\right)_{n \in \mathbb{N}}$, one can extract a subsequence (still denoted $\left(k_{n}\right)_{n \in \mathbb{N}}$ ) converging to a function $\underline{k}_{0} \in \underset{p<N^{\prime}}{\cap} W_{0}^{1, p}(\Omega)$ as $n \rightarrow+\infty$, weakly in each $W_{0}^{1, p}(\Omega)\left(p<N^{\prime}\right)$, strongly in $L^{q}(\Omega)(q<3$ if $N=3, q<+\infty$ if $N=2)$ and almost everywhere in $\Omega$.

In order to conclude, we shall prove the following points:

(a) $u_{0}=\underline{u}_{0}$,

(b) $u_{n} \underset{n \rightarrow+\infty}{\longrightarrow} u_{0}$ strongly in $H_{0}^{1}(\Omega)$,

(c) $k_{0}=\underline{k_{0}}$,

(d) $k_{n} \underset{n \rightarrow+\infty}{\rightarrow} k_{0}$ strongly in $W_{0}^{1, p_{0}}(\Omega)$.

(a) Let $\varphi \in \mathcal{D}(\Omega)$, and consider

$$
I_{n}=\int_{\Omega} \nu_{t}^{n}\left(k_{n}\right) \nabla u_{n} \cdot \nabla \varphi
$$

where

$$
\nu_{t}^{n}\left(k_{n}\right)=1+\ell_{n} a\left(k_{n}\right) .
$$

Because $a(k)$ is a bounded function and $\ell_{n} \underset{n \rightarrow+\infty}{\rightarrow} 0$,

$$
\nu_{t}^{n}\left(k_{n}\right) \underset{n \rightarrow+\infty}{\rightarrow} 1
$$

for instance in $L^{2}(\Omega)$. Then

$$
\nu_{t}^{n}\left(k_{n}\right) \nabla \varphi \underset{n \rightarrow+\infty}{\rightarrow} \nabla \varphi
$$


strongly in $\left(L^{2}(\Omega)\right)^{N}$. Because $\nabla u_{n} \rightarrow \nabla \underline{u}_{0}$ weakly in $\left(L^{2}(\Omega)\right)^{N}$, one deduces that

$$
I_{n} \underset{n \rightarrow+\infty}{\rightarrow} \int_{\Omega} \nabla \varphi \cdot \nabla \underline{u}_{0}
$$

Consequently, $\underline{u}_{0}$ is solution of

$$
\left\{\begin{array}{c}
-\Delta \underline{u}_{0}=f \\
\underline{u}_{0} \in H_{0}^{1}(\Omega) .
\end{array}\right.
$$

The uniqueness of the solution of the Dirichlet problem yields

$$
\underline{u}_{0}=u_{0} .
$$

(b) Strong convergence of the sequence $\left(u_{n}\right)_{n \in \mathbb{N}}$

We shall use the method of "the convergence of the energies" (see $[8,9]$ ). Using $u_{n}$ as test function in the equation satisfied by $u_{n}$ is permitted and yields

$$
\int \nu_{t}^{n}\left(k_{n}\right)\left|\nabla u_{n}\right|^{2}=\int f \cdot u_{n}
$$

On one side, it is clear that

$$
\int_{\Omega} f \cdot u_{n} \underset{n \rightarrow+\infty}{\rightarrow} \int_{\Omega} f \cdot u_{0}
$$

thus,

$$
\int_{\Omega} \nu_{t}^{n}\left(k_{n}\right)\left|\nabla u_{n}\right|^{2} \underset{n \rightarrow+\infty}{\rightarrow} \int_{\Omega} f \cdot u_{0}
$$

On the other hand, one has

$$
\int_{\Omega}\left|\nabla u_{0}\right|^{2}=\int_{\Omega} f \cdot u_{0}
$$

and then

$$
\int_{\Omega} \nu_{t}^{n}\left(k_{n}\right)\left|\nabla u_{n}\right|^{2} \rightarrow \int_{\Omega}\left|\nabla u_{0}\right|^{2}
$$

Applying now Lemma 5.3.4, page 193 in [8], one can conclude that

$$
\sqrt{\nu_{t}^{n}\left(k_{n}\right)} \nabla u_{n} \underset{n \rightarrow+\infty}{\rightarrow} \nabla u_{0}
$$

strongly in $\left(L^{2}(\Omega)\right)^{N}$ and $\left(u_{n}\right)_{n \in \mathbb{N}}$ converges to $u_{0}$ strongly in $H_{0}^{1}(\Omega)$. Moreover,

$$
\nu_{t}^{n}\left(k_{n}\right)\left|\nabla u_{n}\right|^{2} \underset{n \rightarrow+\infty}{\rightarrow}\left|\nabla u_{0}\right|^{2}
$$

strongly in $L^{1}(\Omega)$. 
(c) Arguing as in point (a), and thanks to the boundness of $a(k)$,

$$
\nu_{t}^{n}\left(k_{n}\right) \rightarrow 1
$$

strongly in $L^{p_{0}^{\prime}}(\Omega)$. Thus

$$
\nu_{t}^{n}\left(k_{n}\right) \nabla \varphi \underset{n \rightarrow+\infty}{\rightarrow} \nabla \varphi
$$

strongly in $L^{p_{0}^{\prime}}(\Omega)$. Because $\nabla k_{n} \rightarrow \nabla \underline{k}_{0}$ weakly in $L^{p_{0}}(\Omega)$,

$$
\int_{\Omega} \nu_{t}^{n}\left(k_{n}\right) \nabla k_{n} \cdot \nabla \varphi \underset{n \rightarrow+\infty}{\rightarrow} \int_{\Omega} \nabla \underline{k}_{0} \cdot \nabla \varphi
$$

Applying the result of point (b) yields

$$
\int_{\Omega} \nu_{t}^{n}\left(k_{n}\right)\left|\nabla u_{n}\right|^{2} \varphi \rightarrow \int_{\Omega}\left|\nabla u_{0}\right|^{2} \varphi
$$

Then $\underline{k}_{0}$ is solution of

$$
\left\{\begin{array}{c}
-\Delta \underline{k}_{0}=\left|\nabla u_{0}\right|^{2} \text { in } \mathcal{D}^{\prime}(\Omega) \\
\underline{k}_{0} \in W_{0}^{1, p_{0}}(\Omega)
\end{array}\right.
$$

But because of (15),

$$
\left\|u_{0}\right\|_{H^{2}(\Omega)} \leqslant C\left\|\Delta u_{0}\right\|_{L^{2}}=C\|f\|_{L^{2}},
$$

$C$ being some constant. Consequently, $\left|\nabla u_{0}\right|^{2} \in L^{q}(\Omega), q<3$ if $N=3, q<+\infty$ if $N=2$. In any cases, $\left|\nabla u_{0}\right|^{2} \in L^{2}(\Omega)$. Thus, $\underline{k}_{0} \in H_{0}^{1}(\Omega) \cap H^{2}(\Omega)$ and then $k_{0}=\underline{k}_{0}$.

(d) Strong convergence of $\left(k_{n}\right)_{n \in \mathbb{N}}$

Let $T_{q}$ be the truncature function at height $q$ :

$$
\begin{aligned}
& T_{q}(X)=X \text { if }|X| \leqslant q, \\
& T_{q}(X)=q \operatorname{sgn}(X) \text { if }|X|>q .
\end{aligned}
$$

Applying Lemma 5.3.2, page 195 in [8] to this situation, we obtain

$$
T_{q}\left(k_{n}\right) \underset{n \rightarrow+\infty}{\rightarrow} T_{q}\left(k_{0}\right), \quad \forall q \in \mathbb{R}_{+}^{*},
$$

strongly in $H_{0}^{1}(\Omega)$. Note that this kind of convergence result for the truncatures was first obtained by P.-L. Lions and F. Murat (private communication). In [8], the proof is performed in a very general context and is rather technical. In this context, there is a simplified proof, left to the reader.

Notice also that one has obviously by maximum principle $k_{n} \geqslant 0, k_{0} \geqslant 0$, a.e. in $\Omega$.

Consider now

$$
U_{q}^{n}=\left\{k_{n} \geqslant q\right\} \cup\left\{k_{0} \geqslant q\right\}
$$

One clearly has

$$
\operatorname{mes}\left\{k_{0} \geqslant q\right\} \underset{q \rightarrow+\infty}{\rightarrow} 0 \text {. }
$$


On the other hand, one has

$$
q \operatorname{mes}\left\{k_{n} \geqslant q\right\} \leqslant \int_{\left\{k_{n} \geqslant q\right\}} k_{n} \leqslant C,
$$

$C$ being a constant. The last bound is satisfied because $k_{n} \rightarrow k_{0}$ in $L^{1}(\Omega)$.

Thus,

$$
\operatorname{mes}\left\{k_{n} \geqslant q\right\} \underset{q \rightarrow+\infty}{\rightarrow} 0
$$

uniformely with respect to $n$, and consequently

$$
\operatorname{mes} U_{q}^{n} \underset{q \rightarrow+\infty}{\rightarrow} 0
$$

uniformely with respect to $n$. Consider

$$
I_{n}=\int_{\Omega}\left|\nabla k_{n}-\nabla k_{0}\right|^{p_{0}}
$$

One has

$$
I_{n}=\int_{U_{q}^{n}}\left|\nabla k_{n}-\nabla k_{0}\right|^{p_{0}}+\int_{U_{q}^{n c}}\left|\nabla k_{n}-\nabla k_{0}\right|^{p_{0}}
$$

where

$$
U_{q}^{n c}=\left\{k_{n}<q\right\} \cap\left\{k_{0}<q\right\}
$$

Notice that

$$
\int_{U_{q}^{n c}}\left|\nabla k_{n}-\nabla k_{0}\right|^{p_{0}}=\int_{U_{q}^{n c}}\left|\nabla T_{q}\left(k_{n}\right)-\nabla T_{q}\left(k_{0}\right)\right|^{p_{0}}
$$

On one hand, one has for $p_{0}<p_{1}<N^{\prime}$, by Hölder inequality

$$
\int_{U_{q}^{n}}\left|\nabla k_{n}-\nabla k_{0}\right|^{p_{0}} \leqslant\left(\operatorname{mes} U_{q}^{n}\right)^{p_{0} /\left(p_{1}-p_{0}\right)}\left(\int_{U_{q}^{n}}\left|\nabla k_{n}-\nabla k_{0}\right|^{p_{1}}\right)^{p_{0} / p_{1}}
$$

ad thanks to $(13)$,

$$
\left(\int_{U_{q}^{n}}\left|\nabla k_{n}-\nabla k_{0}\right|^{p_{1}}\right)^{p_{0} / p_{1}} \leqslant C
$$

where $C$ is a constant. Thus,

$$
\int_{U_{q}^{n}}\left|\nabla k_{n}-\nabla k_{0}\right|^{p_{0}} \leqslant C\left(\operatorname{mes} U_{q}^{n}\right)^{p_{0} /\left(p_{1}-p_{0}\right)} .
$$

Let $\varepsilon>0$. There exists $q_{0}$ such that $\forall q \geqslant q_{0}, \forall n \in \mathbb{N}$,

$$
C\left(\operatorname{mes} U_{q}^{n}\right)^{p_{0} /\left(p_{1}-p_{0}\right)} \leqslant \varepsilon .
$$


Thus, $\forall q \geqslant q_{0}, \forall n \in \mathbb{N}$,

$$
\int_{U_{q}^{n}}\left|\nabla k_{n}-\nabla k_{0}\right|^{p_{0}} \leqslant \varepsilon
$$

On the other hand, because of the convergence of truncatures, and $p_{0}<N^{\prime} \leqslant 2$, there exists $n_{0}$ such that $\forall n \geqslant n_{0}$,

$$
\int_{U_{q_{0}}^{n c}}\left|\nabla k_{n}-\nabla k_{0}\right|^{p_{0}}=\int_{U_{q_{0}}^{n c}}\left|\nabla T_{q_{0}}\left(k_{n}\right)-\nabla T_{q_{0}}\left(k_{0}\right)\right|^{p_{0}} \leqslant \varepsilon .
$$

Thus, $\forall n \geqslant n_{0}$, one has $I_{n} \leqslant 2 \varepsilon$, that means

$$
k_{n} \underset{n \rightarrow+\infty}{\rightarrow} k_{0}
$$

strongly in $W_{0}^{1, p_{0}}(\Omega)(*)$, and the proof of the lemma is complete, the corollary being obvious.

$\left(^{*}\right)$ The possible limit being unique, all the sequence converges by a standard compactness argument.

Theorem 3.2. There exists $\ell_{0}>0$ such that for $0<\ell<\ell_{0}$, system $\left(S_{2}\right)$ admits a unique solution.

Proof. We shall use the implicit function theorem combined with Lemma 3.1. Let

$$
\mathcal{Y}=H^{-1}(\Omega) \times W^{-1, p_{0}}(\Omega) .
$$

Notice that $\forall x=(u, k) \in \mathcal{X}$,

$$
-\nabla \cdot\left(\nu_{t}(k) \nabla u\right) \in H^{-1}(\Omega)
$$

$\nu_{t}$ being a bounded function. Thus

$$
\left|\left\langle-\nabla \cdot\left(\nu_{t}(k) \nabla u\right), v\right\rangle\right|=\left|\int \nu_{t}(k) \nabla u . \nabla v\right| \leqslant\left\|\nu_{t}\right\|_{\infty}\|u\|_{H_{0}^{1}}\|v\|_{H_{0}^{1}} .
$$

For the same reason,

$$
-\nabla \cdot\left(\nu_{t}(k) \nabla k\right) \in W^{-1, p_{0}}(\Omega)=\left(W_{0}^{1, p_{0}^{\prime}}(\Omega)\right)^{\prime}
$$

Furthermore, $p_{0}<N^{\prime}$ implies $p_{0}^{\prime}>N$; hence

$$
W_{0}^{1, p_{0}^{\prime}}(\Omega) \subset L^{\infty}(\Omega)
$$

and one has naturally

$$
L^{1}(\Omega) \subseteq W^{-1, p_{0}}(\Omega),
$$

where, for $f \in C^{1}(\Omega)$,

$$
|\langle f, v\rangle|=\left|\int f . v\right| \leqslant\|f\|_{L^{1}}\|v\|_{L^{\infty}} \leqslant C\|f\|_{L^{1}}\|v\|_{W_{0}^{1, p_{0}^{\prime}}} .
$$


Thus it makes sense to introduce

$$
\begin{gathered}
F: \mathbb{R} \times \mathcal{X} \rightarrow \mathcal{Y} \\
(\ell, x) \rightarrow\left(-\nabla \cdot\left(\nu_{t}^{\ell}(k) \nabla u\right)-f,-\nabla \cdot\left(\nu_{t}^{\ell}(k) \nabla k\right)-\nu_{t}^{\ell}(k)|\nabla u|^{2}\right),
\end{gathered}
$$

where $\nu_{t}^{\ell}(k)=1+\ell a(k)$.

Solving system $\left(S_{2}\right)$ is equivalent to solve the equation

$$
F(\ell, x)=0, \quad(x=(u, k)),
$$

positiveness of $k$ being guarantied by the maximum principle.

It is easy to check that $F$ is of class $C^{1}$ (for this reason, $a(k)$ needs to be $\left.C^{1}\right)$.

The couple $x_{0}=\left(u_{0}, k_{0}\right)$ being the unique solution of system $\left(S_{0}\right)$, we have

$$
F\left(0, x_{0}\right)=0
$$

It is also easy to check that

$$
\frac{\partial F}{\partial x}\left(0, x_{0}\right)(\theta, \xi)=\left(-\Delta \theta,-\Delta \xi-2 \nabla u_{0} . \nabla \theta\right)
$$

It can be derived from the classical elliptic theory that $\frac{\partial F}{\partial x}\left(0, x_{0}\right)$ is an isomorphism from $\mathcal{X}$ onto $\mathcal{Y}$.

Applying the implicit function theorem yields that

$$
\begin{aligned}
\exists U & =L \times V \in \mathcal{V}_{\mathbb{R} \times \mathcal{X}}\left(\left(0, x_{0}\right)\right), \\
\exists W & \in \mathcal{V}_{\mathbb{R}}(0), \\
\exists g & \in C^{1}(W, \mathcal{X})
\end{aligned}
$$

such that the following assertions (i) and (ii) are equivalent.

$$
\begin{aligned}
& \text { (i) } \quad(\ell, x) \in U \text { and } F(\ell, x)=0 . \\
& \text { (ii) } \ell \in W \text { and } x=g(\ell) .
\end{aligned}
$$

Now let $\varepsilon_{0}$ be such that

$$
]-\varepsilon_{0}, \varepsilon_{0}[\subseteq L \cap W .
$$

Notice that in the previous considerations only non negative values of $\ell$ were considered, but the mathematical problem posed by system $\left(S_{2}\right)$ is consistent for all $\ell \in \mathbb{R}$ as soon as $|\ell| \leqslant \frac{\rho}{\|a\|_{\infty}}$, for some constant $\rho<1$. Then in the following we shall assume that $\varepsilon_{0} \leqslant \rho /\|a\|_{\infty}$.

Applying now Corollary 3.1, there exists $\varepsilon_{1}>0$ such that $\left.\forall \ell \in\right]-\varepsilon_{1}, \varepsilon_{1}[$,

$$
A_{\ell} \subseteq V
$$

Set $\ell_{0}=\min \left(\varepsilon_{0}, \varepsilon_{1}\right)$.

Let $\ell$ with $|\ell|<\ell_{0}$, and $x_{1}, x_{2}$ both in $A_{\ell}$, then one has

$$
F\left(\ell, x_{1}\right)=0=F\left(\ell, x_{2}\right)
$$


and $x_{1} \in V, x_{2} \in V$.

Thus, $x_{1}=g(\ell)=x_{2}$ and then there is exactly one element in each $A_{\ell}$ for $|\ell|<\ell_{0}$, and Theorem 3.2 is proved.

\subsection{Case of large values of $\ell$}

We now come back to the case $\nu_{t}=1+\ell k^{1 / 2}$ and denote by $\left(u_{\ell}, k_{\ell}\right)$ any solution of system $\left(\mathcal{S}_{2}\right)$.

Theorem 3.3. The sequence $\left(k_{\ell}\right)_{\ell>0}$ converges towards zero when $\ell \rightarrow+\infty$ weakly in $W_{0}^{1, p}(\Omega)$ $\left(\forall p<N^{\prime}\right)$ and strongly in $L^{q}(\Omega), \forall q<3$ if $N=3, \forall q<+\infty$ if $N=2$.

Proof. Thanks to estimate $(13)$, we can extract from $\left(k_{\ell}\right)_{\ell \in \mathbb{R}^{+}}$a subsequence (still denoted by $\left.\left(k_{\ell}\right)_{\ell \in \mathbb{R}^{+}}\right)$such that

$$
k_{\ell} \underset{\ell \rightarrow \infty}{\rightarrow} k_{\infty}\left\{\begin{array}{l}
\text { weakly in } W_{0}^{1, p}(\Omega), \\
\text { strongly in } L^{q}(\Omega), \text { almost everywhere, } q<p^{*}
\end{array}\right.
$$

and this holds for every $p<N^{\prime}, N^{\prime}=3 / 2$ in the case $N=3$ (see [8] Cor. 5.3.1, page 190).

Let $\phi \in C_{c}^{\infty}(\Omega)$. By definition, one has

$$
\int_{\Omega} \nabla k_{\ell} \cdot \nabla \phi+\ell \int_{\Omega} k_{\ell}^{1 / 2} \nabla k_{\ell} \cdot \nabla \phi=\int_{\Omega} \nu_{t}\left(k_{\ell}\right)\left|\nabla u_{\ell}\right|^{2} \phi .
$$

Because of estimate (12), one can extract an other subsequence, identically denoted, such that

$$
\nu_{t}\left(k_{\ell}\right)\left|\nabla u_{\ell}\right|^{2} \underset{\ell \rightarrow+\infty}{\rightarrow} \mu
$$

vaguely in the sense of the measures.

Then

$$
\int \nu_{t}\left(k_{\ell}\right)\left|\nabla u_{\ell}\right|^{2} \phi \underset{\ell \rightarrow+\infty}{\rightarrow}\langle\mu, \phi\rangle
$$

On the other hand,

$$
\int \nabla k_{\ell} \cdot \nabla \phi \rightarrow \int \nabla k_{\infty} \cdot \nabla \phi
$$

It follows from (16) that $\left(\ell \int k_{\ell}^{1 / 2} \nabla k_{\ell} . \nabla \phi\right)$ remains bounded when $\ell \rightarrow+\infty$. Hence

$$
\int_{\Omega} k_{\ell}^{1 / 2} \nabla k_{\ell} \cdot \nabla \phi \underset{\ell \rightarrow+\infty}{\rightarrow} 0
$$

In the case $N=3$ we have $N^{*}=3$. Then

$$
k_{\ell}^{1 / 2} \underset{\ell \rightarrow+\infty}{\rightarrow} k_{\infty}^{1 / 2} \text { strongly in } L^{p}(\Omega), p<6
$$

On the other hand, $N^{\prime}=3 / 2$ and

$$
\nabla k_{\ell} \rightarrow \nabla k_{\infty} \text { weakly in } L^{q}(\Omega), q<3 / 2
$$


Because $\frac{2}{3}+\frac{1}{6}=\frac{5}{6}<1, \quad(17)$ leads to

$$
\int_{\Omega} k_{\ell}^{1 / 2} \nabla k_{\ell} \cdot \nabla \phi \rightarrow \int_{\Omega} k_{\infty}^{1 / 2} \nabla k_{\infty} \cdot \nabla \phi=0 .
$$

Hence, $k_{\infty}$ is solution of the problem

$$
\begin{cases}-\nabla \cdot\left(k_{\infty}^{1 / 2} \nabla k_{\infty}\right)=0=-\Delta\left(k_{\infty}^{3 / 2}\right) & \text { in } \Omega \\ k_{\infty}=0 & \text { on } \partial \Omega .\end{cases}
$$

Moreover, it is easy to verify that $k^{3 / 2} \in \underset{p<6 / 5}{\cap} W_{0}^{1, p}$, and $-\Delta$ is an isomorphism between $W_{0}^{1, p}$ and $W^{-1, p^{\prime}}$ (see [11]). We conclude from (16) that $k_{\infty}=0$. Finally, the limit being unique, all the sequence converges towards 0 which achieves the proof of the theorem.

Proposition 3.1. Define $\nu_{t}^{\ell}=1+\ell k_{\ell}^{1 / 2}$. Let $B \subset \subset \Omega$ be any open set. Then the sequence $\left(\nu_{t}^{\ell}\right)_{\ell \in \mathbb{R}^{+}}$cannot converge almost everywhere in $B$ to a function $\nu_{t}^{\infty} \in L^{q}(B), q>3$, unless $f \equiv 0$ on $B$.

Proof. Assume that there exists an open set $B \subset \subset \Omega$ and a function $\nu_{t}^{\infty} \in L^{q}(B)(q>3)$, such that

$$
\nu_{t}^{\ell} \underset{\ell \rightarrow+\infty}{\rightarrow} \nu_{t}^{\infty} \text { almost everywhere in } B \text {. }
$$

Applying estimate (13), we know that $\nu_{t}^{\ell}$ is bounded in $L^{p}(\Omega)$ for $p<6$. Then for $p<\operatorname{Inf}(q, 6), B$ being bounded

$$
\nu_{t}^{\ell} \underset{\ell \rightarrow+\infty}{\rightarrow} \nu_{t}^{\infty} \text { strongly in } L^{p}(B)
$$

This result is rather classical in the folklore of PDE, and is obtained by combining Egorov theorem with Hölder inequality (see [14]). The details are left to the reader.

Let $\phi \in C_{c}^{\infty}(B)$. One has

$$
\int_{B} \nu_{t}^{\ell} \nabla k_{\ell} \cdot \nabla \phi=\int \nu_{t}^{\ell}\left|\nabla u_{\ell}\right|^{2} \phi
$$

We know by Theorem 3.3 that $\nabla k_{\ell} \rightarrow 0$ in $W_{0}^{1, p}(\Omega)$ weak, for $p<3 / 2$. Because $\nu_{t}^{\ell} \rightarrow \nu_{t}^{\infty}$ strongly in $L^{r}(B), r>$ $3, \nu_{t}^{\ell} \nabla k_{\ell} \rightarrow 0$ weak in $L^{s}(B)$ for some $s>1$. Then, it follows from (19) that

$$
\int_{B} \nu_{t}^{\infty}\left|\nabla u^{\infty}\right|^{2} \phi=0, \quad \forall \phi \in C_{c}^{\infty}(B),
$$

where $u^{\infty}$ is the weak limit in $H_{0}^{1}(B)$ of $u_{\ell}$ when $\ell \rightarrow+\infty$. From $(20)$, one deduces that

$$
\nu_{t}^{\infty}\left|\nabla u^{\infty}\right|^{2}=0 \quad \text { a.e. in } B \text {. }
$$

Moreover one has

$$
\int_{B} \nu_{t}^{\ell} \nabla u_{\ell} \cdot \nabla \phi=\int_{B} f . \phi .
$$

Because of the strong convergence of $\nu_{t}^{\ell}$ to $\nu_{t}^{\infty}$ in $L^{3}(B)$, in particular

$$
\nu_{t}^{\ell} \nabla \phi \underset{\ell \rightarrow+\infty}{\rightarrow} \nu_{t}^{\infty} \nabla \phi
$$


strongly in $L^{2}(B)$. On the other hand, $\nabla u_{\ell} \rightarrow \nabla u^{\infty}$ weakly in $L^{2}(B)$.

Then passing to the limit yields

$$
\int_{B} \nu_{t}^{\infty} \nabla u^{\infty} \cdot \nabla \phi=\int_{B} f \cdot \phi
$$

But, by Cauchy-Schwarz inequality,

$$
\left|\int_{B} \nu_{t}^{\infty} \nabla u^{\infty} \cdot \nabla \phi\right| \leqslant\left(\int_{B} \nu_{t}^{\infty}\left|\nabla u^{\infty}\right|^{2}\right)^{1 / 2}\left(\int_{B} \nu_{t}^{\infty}|\nabla \phi|^{2}\right)^{1 / 2}
$$

and (21) implies that $\int \nu_{t}^{\infty} \nabla u^{\infty} \cdot \nabla \phi=0$, which is compatible with (22) if and only if $f \equiv 0$ on $B$, which concludes the proof.

Proposition 3.2. Let $B \subset \subset \Omega$ an open set, and assume that

$$
\nu_{t}^{\ell} \underset{\ell \rightarrow+\infty}{\rightarrow}+\infty \text { uniformely in } B
$$

then

$$
\int_{B}\left|\nabla u_{\ell}\right|^{2} \underset{\ell \rightarrow+\infty}{\rightarrow} 0
$$

Proof. Towards a contradiction, let us assume that

$$
\exists \delta>0 / \forall \ell_{0} \in \mathbb{R}_{+}^{*}, \exists \ell \geqslant \ell_{0} \text { such that } \int_{B}\left|\nabla u_{\ell}\right|^{2} \geqslant \delta .
$$

Then for $\ell_{0}=n, \exists \ell_{n} \geqslant \ell_{0}$ such that $\int_{B}\left|\nabla u_{\ell_{n}}\right|^{2} \geqslant \delta$.

On the other hand,

$$
\forall M>0, \quad \exists \ell_{0} / \forall x \in B, \quad \forall \ell \geqslant \ell_{0}, \nu_{t}^{\ell} \geqslant M
$$

The consequence is

$$
\int \nu_{t}^{\ell_{n}}\left|\nabla u_{\ell_{n}}\right|^{2} \geqslant M \delta
$$

which states that $\int \nu_{t}^{\ell_{n}}\left|\nabla u_{\ell_{n}}\right|^{2} \underset{\ell \rightarrow+\infty}{\rightarrow}+\infty$, which is in contradiction with estimate (12).

In order to conclude this section, we prove in the following lemma an additional convergence result.

Lemma 3.2. Assume that $\nu_{t}^{\ell}=1+\ell a\left(k_{\ell}\right)$, where $a$ is a continuous function such that $a(0) \neq 0$ and $|a(k)| \leqslant$ $1+k^{\theta}, \theta \in[0,1[$, then

$$
u_{\ell} \underset{\ell \rightarrow \infty}{\rightarrow} 0
$$

weakly in $H_{0}^{1}(\Omega)$, strongly in $L^{q}(\Omega), q<3 / 2$.

Proof. We already have proved that $k_{\ell} \rightarrow 0$ strongly in $L^{q}(\Omega), 1<q<3$, as $\ell \rightarrow \infty$.

Applying the inverse Lebesgue theorem, we can extract from $\left(k_{\ell}\right)$ a subsequence still denoted $\left(k_{\ell}\right)$ such that $k_{\ell} \underset{\ell \rightarrow \infty}{\rightarrow} 0$ a.e. in $\Omega$ and such that there exists $g \in L^{q}(\Omega) \quad(1<q<3)$ satisfying $\left|k_{\ell}\right| \leqslant g$, a.e. in $(\Omega)$.

Then we have the majoration $\left|a\left(k_{\ell}\right)\right| \leqslant 1+g^{\theta}$ in $L^{q / \theta}(\Omega)$. As the function $a$ is continuous, $a\left(k_{\ell}\right) \rightarrow a(0)$ a.e. in $\Omega$. Consequently, applying the Lebesgue dominated convergence theorem, $a\left(k_{\ell}\right) \rightarrow a(0)$ strongly in $L^{2}(\Omega)$ (for $\frac{q}{\theta}=2$, which is possible).

On the other hand $u_{\ell} \underset{\ell \rightarrow \infty}{\rightarrow} u_{\infty}$ weakly in $H_{0}^{1}(\Omega)$.

Let $\phi \in C_{c}^{\infty}(\Omega)$. By definition one has

$$
\int_{\Omega} \nabla u_{\ell} \cdot \nabla \phi+\ell \int_{\Omega} a\left(k_{\ell}\right) \nabla u_{\ell} \cdot \nabla \phi=\int_{\Omega} f \cdot \phi .
$$


Applying the previous convergence results, we can conclude that

$$
\begin{aligned}
\int_{\Omega} \nabla u_{\ell} \cdot \nabla \phi & \rightarrow \int_{\Omega} \nabla u_{\infty} \cdot \nabla \phi \\
\int_{\Omega} a\left(k_{\ell}\right) \nabla u_{\ell} \cdot \nabla \phi & \rightarrow \int_{\Omega} a(0) \nabla u_{\infty} \cdot \nabla \phi .
\end{aligned}
$$

Thus, $\ell \int_{\Omega} a\left(k_{\ell}\right) \nabla u_{\ell} \cdot \nabla \phi$ remains bounded as $\ell \rightarrow \infty$. Consequently, since $a(0) \neq 0$, we have

$$
\int_{\Omega} \nabla u_{\infty} \cdot \nabla \phi=0, \quad \forall \phi \in C_{c}^{\infty}(\Omega),
$$

and therefore $\Delta u_{\infty}=0, u_{\infty} \in H_{0}^{1}(\Omega)$, that is to say $u_{\infty}=0$.

It has to be noted that in this section we have enforced homogeneous Dirichlet conditions on $\partial \Omega$. With only slight modifications, one can prove that the results are still valid for more realistic boundary conditions as those used in Section 4 and more realistic models as for instance model (25) below.

Theoretical results have been obtained for system $\left(\mathcal{S}_{2}\right)$, when the term $\varepsilon=-\frac{1}{\ell} k^{3 / 2}$ is neglected. Except uniqueness theorem, these results are still valid for $\left(\mathcal{S}_{1}\right)$ until we have $\ell>0$.

\section{Numerical RESUlts}

We present in this section two numerical experiments. The first one concerns the scalar model governed by equations $\left(\mathcal{S}_{1}\right)$ and is solved in a three-dimensional domain $\Omega$. The second one is two-dimensional but solves a more complete problem including pressure and vectorial velocity. We use iterative methods to solve these nonlinear problems, but we are not able to prove the convergence of these methods. Similar problems are considered in [2] where strong regularity assumptions are necessary to obtain theoretical convergence results.

\subsection{The scalar model}

We seek for $(u, k)$ solution of the equations

$$
\left\{\begin{array}{l}
-\nabla \cdot\left(\nu_{t} \nabla u\right)=f, \\
-\nabla \cdot\left(\nu_{t} \nabla k\right)=\nu_{t}|\nabla u|^{2}-\frac{1}{\ell} k^{3 / 2} \text { in } \Omega,
\end{array}\right.
$$

where $u$ represents the scalar velocity, $k$ the turbulent kinetic energy. The two equations are coupled by the right-handed term in the second equation and by the expression of the turbulent viscosity $\nu_{t}=1+\ell k^{1 / 2}$. The parameter $\ell$ is interpreted as the mixing length and controls the turbulent part in $\nu_{t}$. We are going to compare the results obtained with different values of $\ell$. We solve the set of equations $\left(\mathcal{S}_{1}\right)$ in the cube $\left.\Omega=\right] 0,1{ }^{3}$. The boundary conditions enforced on $\partial \Omega$ are described hereafter:

- at the surface $\Gamma_{0}$ of equation $z=1$, the flow is driven by a wind-stress $f w$ represented in Figure 1

$$
\nu_{t} \frac{\partial u}{\partial z}=f w
$$

- on the other parts of the boundary, $\Gamma$, we put $u=0$

- the kinetic energy $k$ has to verify

$$
k=0, \text { on } \partial \Omega .
$$




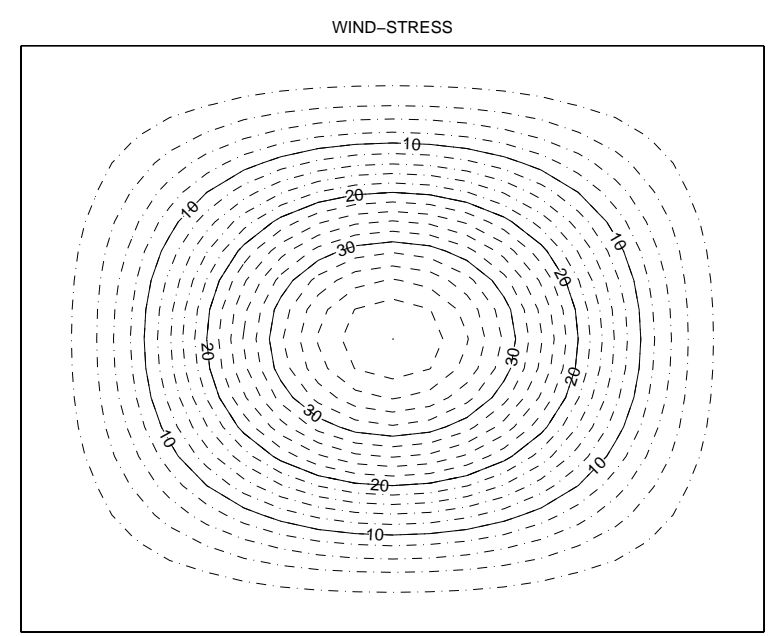

FiguRE 1 .

The results presented in this section have been obtained with a forcing $f=10$.

The weak formulation of problem $\left(\mathcal{S}_{1}\right)$ can be written as follows.

Find $(u, k)$ such that

$$
\left\{\begin{array}{l}
a_{k}(u, v)=(f, v)+\left(f w, \gamma_{o} v\right)_{\Gamma_{0}}, \\
a_{k}(k, w)+e_{k}(k, w)=b_{k}(u, w), \\
\forall v \in \mathcal{V}, \forall w \in \mathcal{W},
\end{array}\right.
$$

where

$$
\begin{gathered}
\mathcal{V}=\left\{v \in H^{1}(\Omega) / v=0 \text { on } \Gamma\right\}, \mathcal{W}=H_{0}^{1}(\Omega), \\
a_{k}(u, v)=\left(\left(1+\ell k^{1 / 2}\right) \nabla u, \nabla v\right), \\
b_{k}(u, w)=\left(\left(1+\ell k^{1 / 2}\right)|\nabla u|^{2}, w\right), \\
e_{k}(k, w)=\frac{1}{\ell}\left(k^{3 / 2}, w\right) .
\end{gathered}
$$

Here, (.,.) denotes the scalar product in $L^{2}(\Omega) ;(,)_{\Gamma_{0}}$ denotes the scalar product in $L^{2}\left(\Gamma_{0}\right)$.

We are going to solve problem (23) by using an iterative algorithm. The couple $\left(u^{n}, k^{n}\right)$ being the solution at step $n,\left(u^{n+1}, k^{n+1}\right)$ has to verify

$$
\left\{\begin{array}{l}
a_{n}\left(u^{n+1}, v\right)=(f, v)+\left(f w, \gamma_{0} v\right)_{\Gamma_{0}}, \\
a_{n}\left(k^{n+1}, w\right)+e_{n}\left(k^{n+1}, w\right)=b_{n}\left(u^{n+1}, w\right), \\
\forall v \in \mathcal{V}, \forall w \in \mathcal{W}
\end{array}\right.
$$


where

$$
\begin{aligned}
& a_{n}\left(u^{n+1}, v\right)=\left(\left(1+\ell\left(k^{n}\right)^{1 / 2}\right) \nabla u^{n+1}, \nabla v\right), \\
& b_{n}\left(u^{n+1}, w\right)=\left(\left(1+\ell\left(k^{n}\right)^{1 / 2}\right)\left|\nabla u^{n+1}\right|^{2}, w\right), \\
& e_{n}\left(k^{n+1}, w\right)=\frac{1}{\ell}\left(\left(k^{n}\right)^{1 / 2} k^{n+1}, w\right) .
\end{aligned}
$$

In order to initialize the iterations we set $k^{0}=0$ and so

$$
\begin{aligned}
& a_{0}\left(u^{1}, v\right)=\left(\nabla u^{1}, \nabla v\right), \\
& b_{0}\left(u^{1}, w\right)=\left(\left|\nabla u^{1}\right|^{2}, w\right), \\
& e_{0}\left(k^{1}, w\right)=0 .
\end{aligned}
$$

Then $\left(u^{1}, k^{1}\right)$ is obtained by solving equations $(24)$.

The cubic domain $\Omega$ is discretized into a regular $10 \times 10 \times 10$ hexaedral mesh. At each iteration $n \rightarrow n+1$ problem (24) is solved by means of finite elements of $Q_{1}$ type, using the finite elements code MELINA (see [12]). Spaces $\mathcal{V}$ and $\mathcal{W}$ are replaced in equations (24) by classical finite elements approximation spaces $\mathcal{V}_{h}$ and $\mathcal{W}_{h}$ (see [7] or [15]).

We discuss below the results obtained for different values of the mixing length $\ell$. For each value of $\ell$, velocity $u$, turbulent kinetic energy $k$ and eddy viscosity $\nu_{t}$ which are plotted in Figures 2 to 13 are the stationary solutions numerically obtained after convergence of the iterative algorithm solving problem (24) at each step.

The isolines of turbulent kinetic energy $k$ are plotted in Figures 2 to 6 for different values of the mixing length, $\ell=0.001,0.01,0.1,1$ and 10. On each figure three sections are plotted corresponding to fixed values of $x: 0.25,0.5$ and 0.75 . We observe that $k$ increases from $k=0$ to a maximum value about 3.5 as $\ell$ increases between 0 and 0.1. Maximum values of $k$ are obtained for $\ell$ between 0.05 and 0.1 . Then $k$ decreases quickly, its maximum being about 1.8 when $\ell=1$ and 0.3 when $\ell=10$. Numerical experiments performed with $\ell$ greater than 10 show that turbulent kinetic energy $k$ tends quickly towards 0 .

The turbulent part $\ell k^{1 / 2}$ of the viscosity coefficient $\nu_{t}$ is plotted in Figures 7 to 10 for $\ell=0.1,1,10$ and 100 . We observe that $\nu_{t}-1$ is an increasing function of the mixing length $\ell$ and that maxima values of $\left(\nu_{t}-1\right)$ are about $0.18,1.2,5$ and 20 respectively for $\ell=0.1,1,10$ and 100 .

We can deduce from the behaviour of $\nu_{t}$ that velocity $u$ is a decreasing function of $\ell$. This is confirmed by Figures 11 to 13 which represent isolines of $u$ for $\ell=0.1,1$ and 10 . For $\ell$ greater than $10, u$ tends quickly to 0 but the shape of isocontours remains unchanged.

The term $\varepsilon=-\frac{1}{\ell} k^{3 / 2}$ appearing in system $\left(\mathcal{S}_{1}\right)$ represents the dissipation due to small scales. If we neglect this term in the numerical model, we observe that the behaviour of the turbulent kinetic energy $k$ is modified only for small values of the mixing length $\ell: k$ is then a decreasing function of $\ell$, its maximum $k_{0}$ being reached for $\ell=0$ when turbulent viscosity vanishes. Comparing the results given by the two different models, with or without $\varepsilon$, we notice that $k$ values are slightly different for $\ell=0.1$ and identical from $\ell=1$. It is obvious that $\varepsilon$ term becomes negligible as $\ell$ increases. However $\varepsilon$ term doesn't affect the behaviour of velocity $u$ even if $\ell$ is small. For values of $\ell$ lower than 0.1 , differences observed on turbulent kinetic energy $k$ induce only small variations of the turbulent viscosity $\ell k^{1 / 2}$. When $\varepsilon$ is neglected, $\nu_{t}$ is slightly larger but variations being about $10^{-2}$ they don't affect significantly the values of velocity $u$. From $\ell=1, k$ and $u$ are exactly the same with or without the dissipative term $\varepsilon$. 


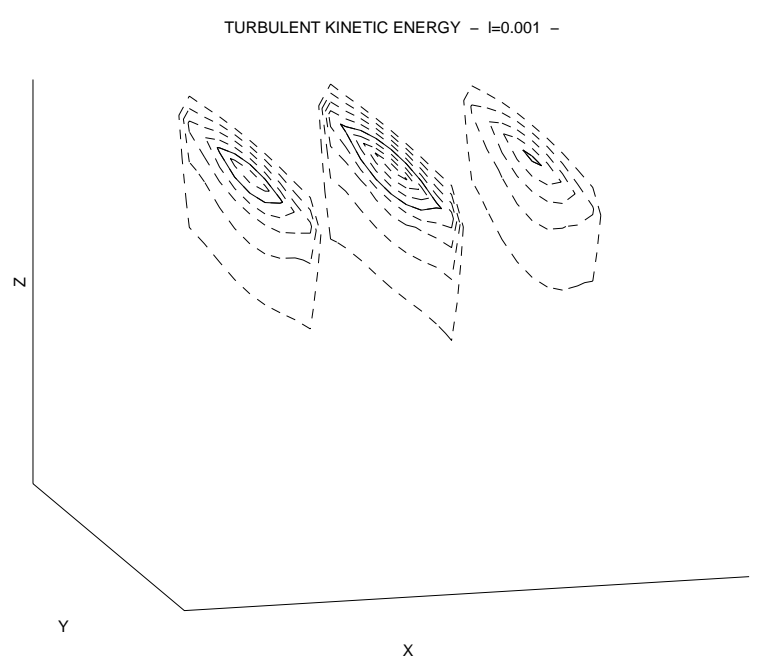

FiguRE 2. Interval between 2 isolines is equal to 0.1 . Solid lines: $k=0.5,1,1.5 \ldots$

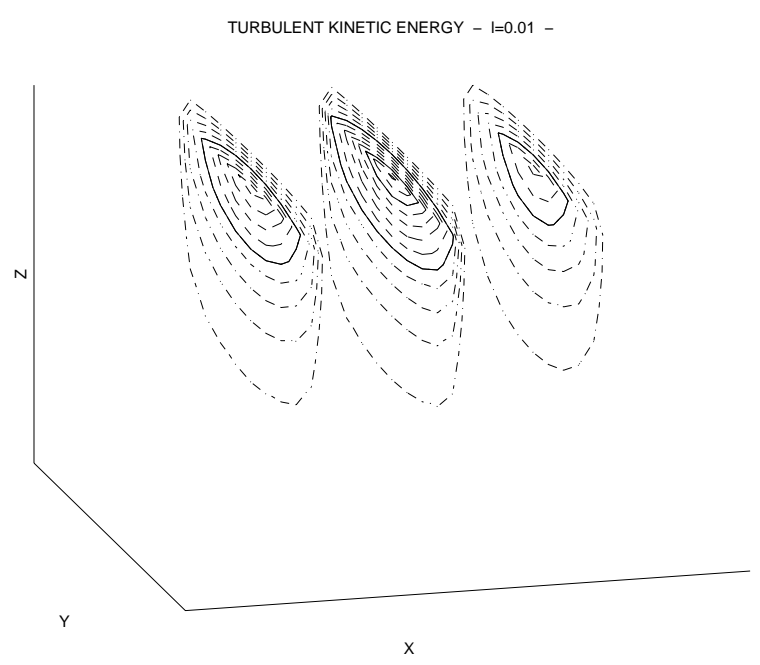

FIGURE 3. Interval between 2 isolines is equal to 0.2 . Solid lines: $k=1,2,3 \ldots$

\subsection{The velocity-pressure model}

We consider in this section a more realistic model including gradient of pressure and continuity equation. The domain $\Omega$ is now the square $] 0,1[\times] 0,1\left[\right.$ in the $(x, z)$ plane. $U=\left(u_{1}, u_{2}\right)$ denotes the vectorial velocity, $p$ the pressure and $k$ the kinetic energy.

We seek for $(U, p, k)$ solution of the following equations

$$
\begin{cases}-\nabla \cdot\left(\nu_{t} \nabla U\right)+\nabla p=F, & (a) \\ \nabla \cdot U=0, & (b) \\ -\nabla \cdot\left(\nu_{t} \nabla k\right)=\nu_{t}|\nabla U|^{2}-\frac{1}{\ell} k^{3 / 2}, & (c)\end{cases}
$$

set in the open set $\Omega$. 


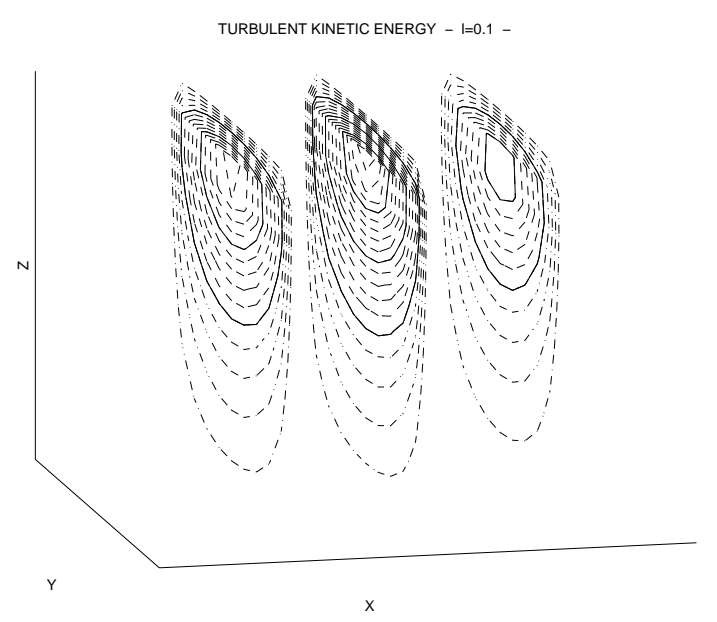

Figure 4. Interval between 2 isolines is equal to 0.2 . Solid lines: $k=1,2,3 \ldots$

TURBULENT KINETIC ENERGY - $\mid=10$ -

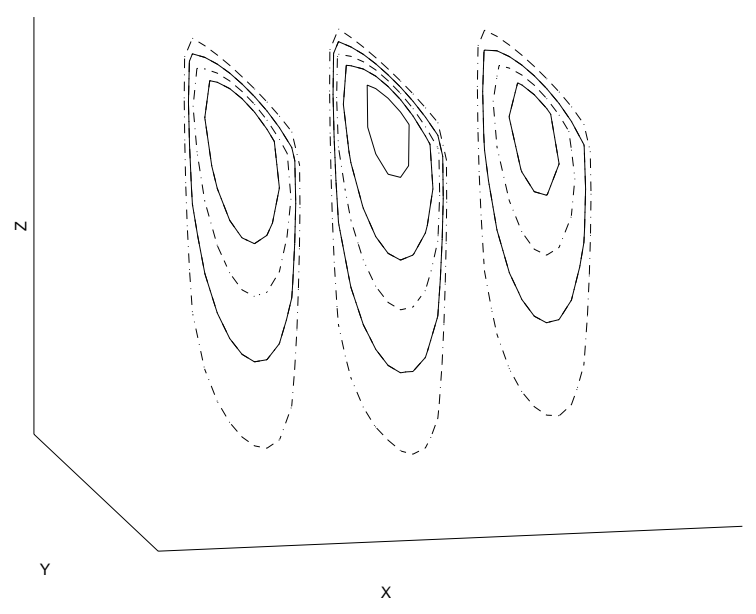

Figure 6. Interval between 2 isolines is equal to 0.05 . Solid lines: $k=0.1,0.2,0.3 \ldots$

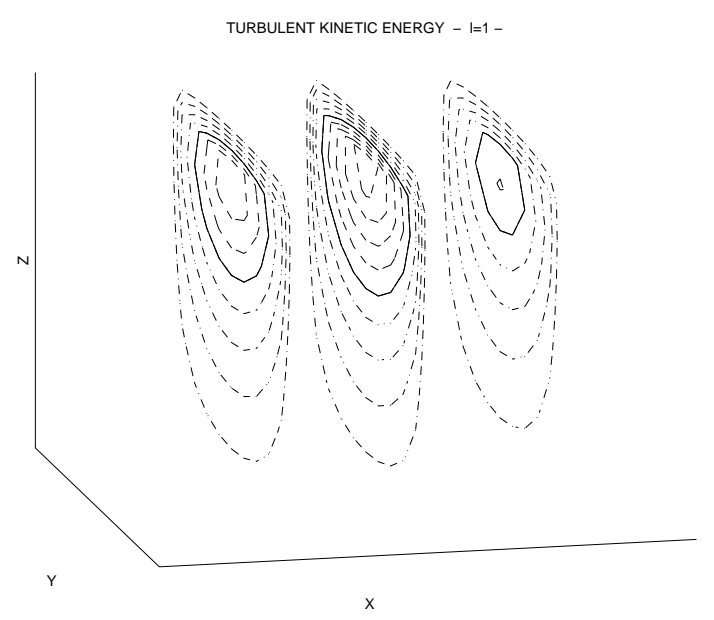

Figure 5. Interval between 2 isolines is equal to 0.2 . Solid lines: $k=1,2,3 \ldots$

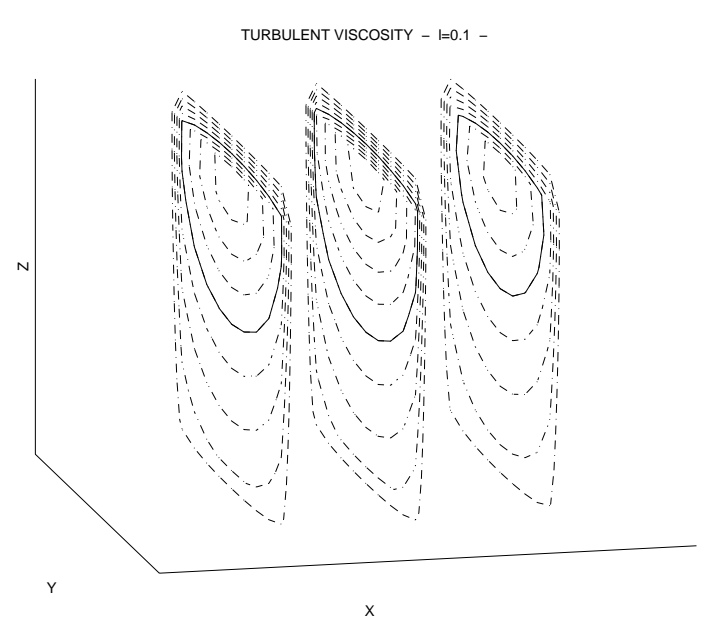

Figure 7. Interval between 2 isolines is equal to 0.02 . Solid lines: $\left(\nu_{t}-1\right)=0.1,0.2,0.3 \ldots$

The boundary conditions enforced on $\partial \Omega$ are described hereafter.

- At the surface $\Gamma_{0}$, the flow is driven by a wind-stress $F W=\left(f w_{1}, 0\right)$,

$$
\nu_{t} \frac{\partial u_{1}}{\partial z}=f w_{1} \text { and } u_{2}=0
$$

- On the other parts of the boundary, $\Gamma$, we impose $U . n=0, \frac{\partial(U . t)}{\partial n}=0$, where $(t, n)$ denotes the unit vectors tangent and normal to $\Gamma$. We note $\Gamma=\Gamma_{1} \cup \Gamma_{2} \cup \Gamma_{3}$ where $\Gamma_{1}$ denotes the bottom of the domain $\Omega, \Gamma_{2}\left(\right.$ resp. $\left.\Gamma_{3}\right)$ its westerly (resp. easterly) boundary. 
TURBUIENT VISCOSITY - I=1. -

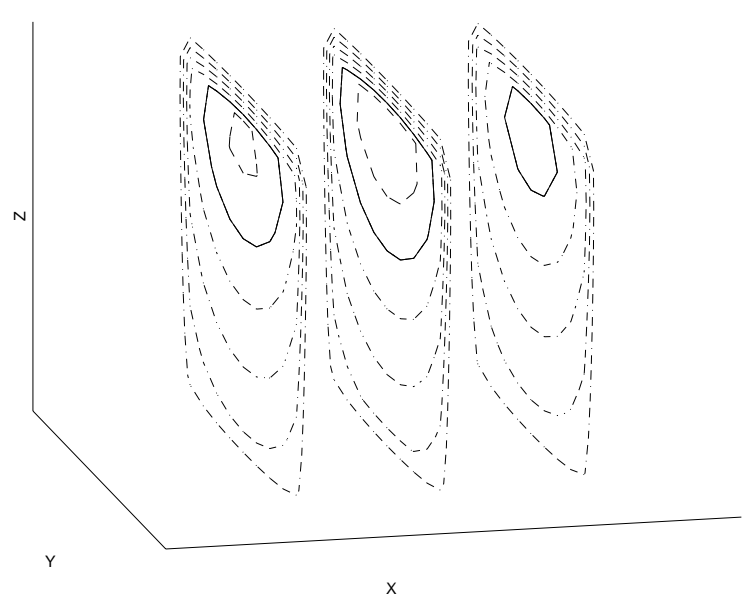

FiguRE 8. Interval between 2 isolines is equal to 0.2. Solid lines: $\left(\nu_{t}-1\right)=1,2,3 \ldots$

TURBULENT VISCOSITY - I=100 -

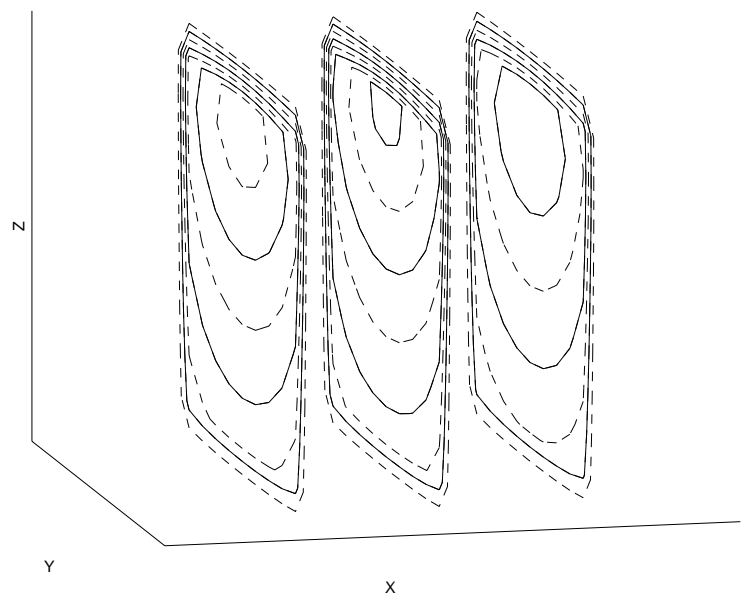

Figure 10. Interval between 2 isolines is equal to 2.5. Solid lines: $\left(\nu_{t}-1\right)=5,10,15 \ldots$
TURBUIENT VISCOSITY - I=10 -

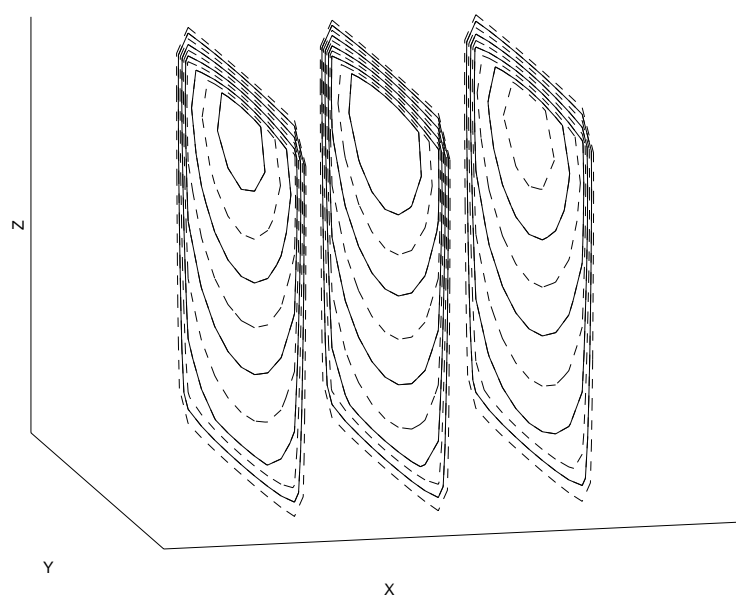

FiguRE 9. Interval between 2 isolines is equal to 0.5. Solid lines: $\left(\nu_{t}-1\right)=1,2,3$.

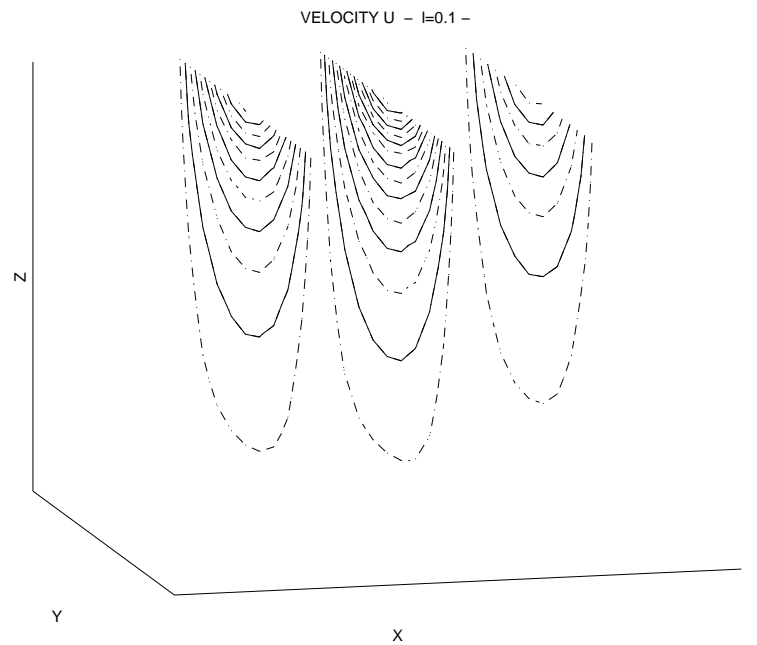

FIGURE 11. Interval between 2 isolines is equal to 0.5 . Solid lines: $u=1,2,3$..

- The turbulent kinetic energy $k$ has to verify $k=0$, on $\partial \Omega$.

The weak formulation of Problem (25) can be written:

find $(U, p, k)$ such that

$$
\left\{\begin{array}{l}
a_{k}(U, V)-d(V, p)=(F, V)+\left(F W, \gamma_{0} V\right)_{\Gamma_{0}} \\
d(U, q)=0 \\
a_{k}(k, w)+e_{k}(k, w)=b_{k}(U, w) \\
\forall V \in \mathcal{V}, \forall w \in \mathcal{W}, \forall q \in L^{2}(\Omega)
\end{array}\right.
$$




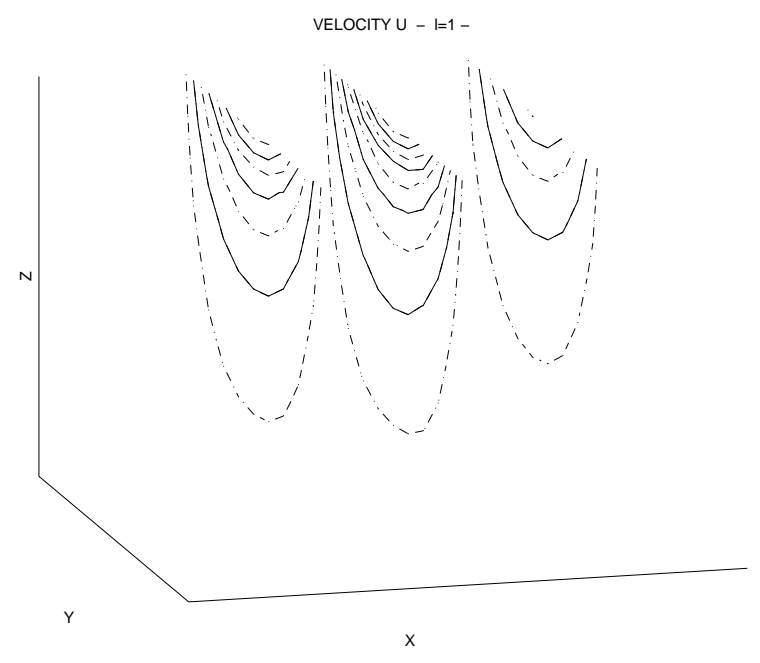

Figure 12. Interval between 2 isolines is equal to 0.5. Solid lines: $u=1,2,3 \ldots$

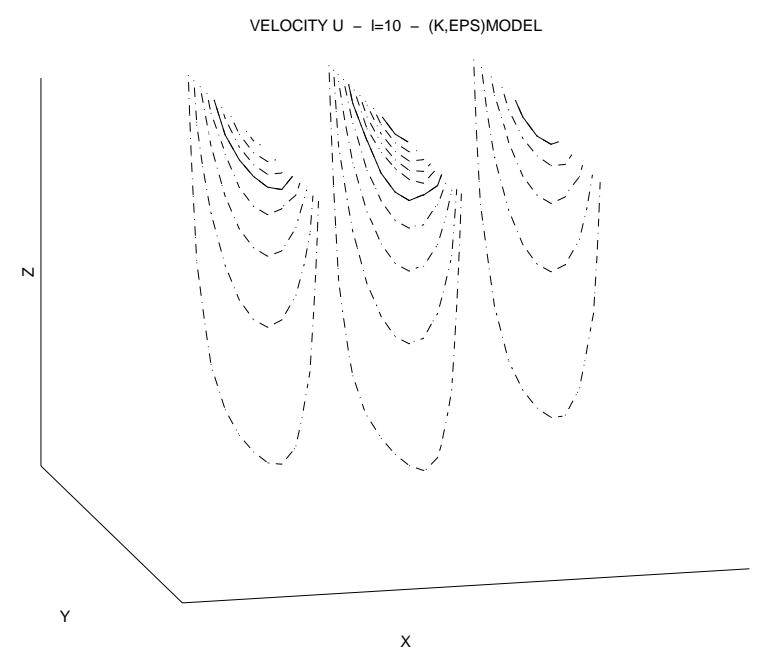

Figure 13. Interval between 2 isolines is equal to 0.2 . Solid lines: $u=1,2,3 \ldots$

where $\mathcal{V}=\left\{V=\left(v_{1}, v_{2}\right) \in\left(H^{1}(\Omega)\right)^{2} / v_{1}=0\right.$ on $\Gamma_{2} \cup \Gamma_{3}, v_{2}=0$ on $\left.\Gamma_{0} \cup \Gamma_{1}\right\}, \mathcal{W}=H_{0}^{1}(\Omega)$,

$$
\begin{aligned}
& a_{k}(U, V)=\left(\left(1+\ell k^{1 / 2}\right) \nabla U, \nabla V\right), \\
& d(U, q)=(\nabla \cdot U, q), \\
& b_{k}(U, w)=\left(\left(1+\ell k^{1 / 2}\right)|\nabla U|^{2}, w\right), \\
& e_{k}(k, w)=\frac{1}{\ell}\left(k^{3 / 2}, w\right) .
\end{aligned}
$$

Problem (26) is solved by using the same iterative algorithm as in the previous section. The triplet $\left(U^{n}, p^{n}, k^{n}\right)$ denotes the solution at step $n$. The solution $\left(U^{n+1}, p^{n+1}, k^{n+1}\right)$ at step $n+1$ has to verify the following problem

$$
\left\{\begin{array}{l}
a_{n}\left(U^{n+1}, V\right)-d\left(V, p^{n+1}\right)=(F, V)+\left(F W, \gamma_{0} V\right)_{\Gamma_{0}}, \\
d\left(U^{n+1}, q\right)=0 \\
a_{n}\left(k^{n+1}, w\right)+e_{n}\left(k^{n+1}, w\right)=b_{n}\left(U^{n+1}, w\right) \\
\forall V \in \mathcal{V}, \forall w \in \mathcal{W}, \forall q \in L^{2}(\Omega)
\end{array}\right.
$$

where

$$
\begin{aligned}
& a_{n}\left(U^{n+1}, V\right)=\left(\left(1+\ell\left(k^{n}\right)^{1 / 2}\right) \nabla U^{n+1}, \nabla V\right), \\
& b_{n}\left(U^{n+1}, w\right)=\left(\left(1+\ell\left(k^{n}\right)^{1 / 2}\right)\left|\nabla U^{n+1}\right|^{2}, w\right), \\
& e_{n}\left(k^{n+1}, w\right)=\frac{1}{\ell}\left(\left(k^{n}\right)^{1 / 2} k^{n+1}, w\right) .
\end{aligned}
$$

We search for $U^{n+1} \in \mathcal{V}, k^{n+1} \in \mathcal{W}, p^{n+1} \in \mathcal{M}$ where $\mathcal{M}=\left\{q \in H^{1}(\Omega) / \frac{1}{\operatorname{mes}\left(\Gamma_{0}\right)} \int_{\Gamma_{0}} q=K\right\}, K$ being a given constant. Definition of space $\mathcal{M}$ allows to obtain a unique pressure $p^{n+1}$ for each velocity $U^{n+1}$.

The pair $\left(U^{n+1}, p^{n+1}\right)$ verifies the mixed velocity-pressure Problem (27.a and $\left.b\right)$. We are going to solve numerically this problem by means of a mixed finite-elements method. The square domain $\Omega$ is discretized into a regular $m \times m$ quadrangular mesh and we will use the $Q_{2} / Q_{1}$ finite element: Lagrange interpolation 
of degree 2 is used for velocity and interpolation of degree 1 for pressure. This finite element verifies the Ladyzenskaya-Brezzi-Babuska compatibility condition (see in [7]).

Space discretization of the mixed velocity-pressure Problem leads to such a linear system

$$
\begin{array}{|c|c|}
\hline A_{n} & -D^{T} \\
\hline D & 0 \\
\hline p^{n+1}
\end{array} \begin{array}{|c|}
\hline U^{n+1} \\
\hline 0 \\
\hline
\end{array}
$$

The approximated solution is still denoted by $\left(U^{n+1}, p^{n+1}\right)$ and we search for $\left(U^{n+1}, p^{n+1}\right) \in \mathcal{V}_{h} \times \mathcal{M}_{h}$. The space $\mathcal{V}_{h}$ (resp. $\mathcal{M}_{h}$ ) is the classical space approximating $\mathcal{V}$ (resp. $\mathcal{M}$ ) when using a finite element of $Q_{2}$ (resp. $\left.Q_{1}\right)$ type $([7,15])$. We describe hereafter the algorithm of resolution of (28) (see [1]).

$A_{n}$ is a positive defined matrix of order $M=2(2 m+1)^{2}$ and System (28) can be written

$$
\begin{aligned}
D\left(A_{n}\right)^{-1} D^{T} p^{n+1} & =-D\left(A_{n}\right)^{-1} G \\
A_{n} U^{n+1} & =G+D^{T} p^{n+1} .
\end{aligned}
$$

It can be easily verified that $D A_{n}^{-1} D^{T}$ is a symmetric, positive defined matrix. Therefore System (29) (30) has one unique solution $\left(U^{n+1}, p^{n+1}\right)$.

We first compute $p^{n+1}$ using a conjugated gradient method and then $U^{n+1}$ is obtained by solving (30). The algorithm for calculus of pressure is described hereafter. The main difficulty results from the fact that matrix $A_{n}^{-1}$ is unknown.

\section{Step 1: Initialization}

- $p_{0}$ given

- $U_{0}$ solution of $A_{n} U_{0}=G+D^{T} p_{0}$

- residual $R_{0}=-D U_{0}$

- descent direction $S_{0}=R_{0}$.

\section{Step $i \rightarrow i+1$}

- $p_{i}, R_{i}, S_{i}$ are known

- $X_{i}$ solution of $A_{n} X_{i}=D^{T} S_{i}$

- $p_{i+1}=p_{i}+\beta_{i} S_{i}$, where $\beta_{i}=\frac{\left(S_{i}, R_{i}\right)}{\left(S_{i}, D X_{i}\right)}$

- residual $R_{i+1}=R_{i}-\beta_{i} D X_{i}$

- descent direction $S_{i+1}=R_{i+1}+\gamma_{i} S_{i}$, where $\gamma_{i}=-\frac{\left(D X_{i}, R_{i+1}\right)}{\left(D X_{i}, R_{i}\right)}$.

Equation (27.c) satisfied by the kinetic energy $k^{n+1}$ is discretized using $Q_{2}$ finite elements and leads to the linear system:

$$
\left(\tilde{A}_{n}+E_{n}\right) k^{n+1}=B\left(k^{n}, U^{n+1}\right)=B_{n+1 / 2}
$$


The complete algorithm for solving Problem (27) is then the following

$$
\begin{aligned}
& \left\{\begin{array}{c}
\text { Initialization: } \\
k^{0}=0, \nu^{0}=1 \rightarrow \text { matrices } A_{0}, \tilde{A}_{0}
\end{array}\right. \\
& \downarrow \\
& \text { Step } 1\left\{\begin{array}{c}
\text { Conjugated gradient } \rightarrow p^{1} \\
A_{0} U^{1}=G+D^{T} p^{1} \rightarrow U^{1} \\
\tilde{A}_{0} k^{1}=B_{1 / 2} \rightarrow k^{1}
\end{array}\right. \\
& \downarrow \\
& \left\{\begin{array}{c}
P^{n}, U^{n}, k^{n}: \text { computed at step } n \\
\text { Viscosity } \nu^{n}=1+\ell\left(k^{n}\right)^{1 / 2} \rightarrow A_{n}, \tilde{A}_{n}
\end{array}\right. \\
& \downarrow \\
& \text { Step }(n+1)\left\{\begin{array}{c}
\text { Conjugated gradient } \rightarrow p^{n+1} \\
A_{n} U^{n+1}=G+D^{T} p^{n+1} \rightarrow U^{n+1} \\
\left(\tilde{A}_{n}+E_{n}\right) k^{n+1}=B_{n+1 / 2} \rightarrow k^{n+1}
\end{array}\right. \\
& \downarrow
\end{aligned}
$$

Convergence test.

The numerical discretization uses the finite elements code MELINA (see [12]). Results described in this section have been obtained with a body force $F=0$. The flow is driven by a wind-stress $F W=\left(f w_{1}, 0\right)$ plotted in Figure 14. Figures discussed hereafter represent the approximated solutions obtained for different values of the mixing length $\ell$ after convergence of the iterative algorithm previously described.

The isolines of turbulent kinetic energy $k$ are plotted in Figures 15 to 19 for the following values of the mixing length $\ell=0.01,0.05,0.1,1,10$. The behaviour of $k$ is the same as in the three-dimensional scalar model. For $\ell=0$ equation (25.c) degenerates and its solution is $k=0$. For $\ell$ varying between 0 and $0.1, k$ is an increasing function of $\ell$. Then $k$ decreases and tends quickly towards 0 for values of $\ell$ greater than 10 .

Turbulent viscosity $\left(\nu_{t}-1\right)=\ell k^{1 / 2}$ is plotted in Figures 20 to 23 for $\ell=0.1,1,10$ and $100 .\left(\nu_{t}-1\right)$ is an increasing function of $\ell$ : its maxima values are about $0.2,1.5,5.5$ and 20 respectively for $\ell=0.1,1,10$ and 100 .

For any values of $\ell$, velocity field $U=\left(u_{1}, u_{2}\right)$ has always the same eddy shape represented in Figure 24 but due to the variations of $\nu_{t},|U|$ is a decreasing function of $\ell$. Isocontours of $|U|$ are plotted in Figures 25 to 27 for $\ell=0.1,1$ and 10 .

Pressure $p$ is plotted in Figure 28 for $\ell=0.1$. The profile of $p$ is very stable and is only weakly modified as $\ell$ varies.

As in the previous case, we have tested the impact of the dissipative term $\varepsilon=-\frac{1}{\ell} k^{3 / 2}$. If $\varepsilon$ is neglected, the behaviour of $k$ is modified for $\ell$ lower that 1 but the impact on turbulent viscosity $\ell k^{1 / 2}$ remains small. Figures 20 and 29 represent $\left(\nu_{t}-1\right)$ for $\ell=0.1$ respectively when dissipation $\varepsilon$ is present or neglected. The presence of $\varepsilon$ term reduces maxima values of $\nu_{t}$ from 1.22 to 1.19 . Such a difference about $3.10^{-2}$ on viscosity coefficient gives rise to very small variations on velocity. When $\ell$ is greater than 0.1 , we obtain exactly the same results in the two cases which is natural since $\varepsilon$ becomes negligible as $\ell$ is large enough.

We have studied two types of numerical models: the first one is three-dimensional and solves a simple scalar model, the second one is two dimensional but solves a more complicated set of equations with pressure and vectorial velocity. In the two cases and for any values of the mixing length $\ell$, we obtain regular solutions as well for turbulent kinetic energy $k$ as for velocity and pressure. This backs up the conjecture of uniqueness 


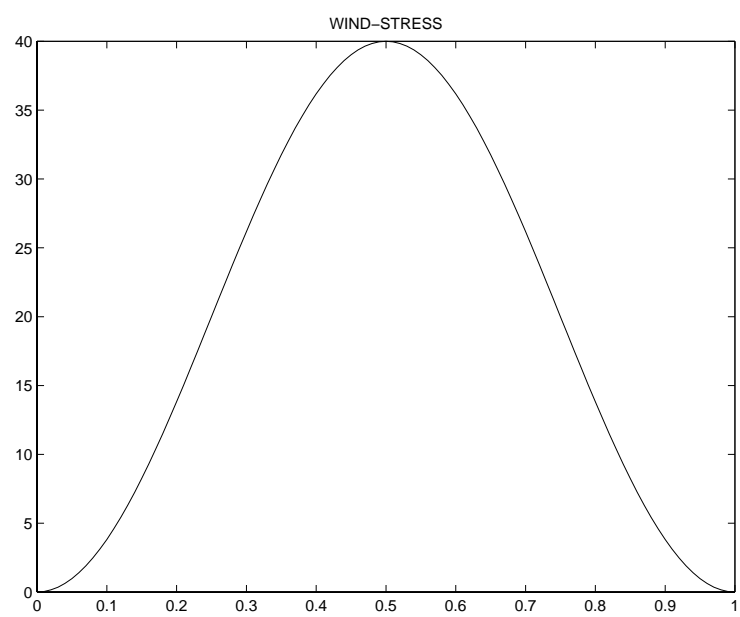

Figure 14.

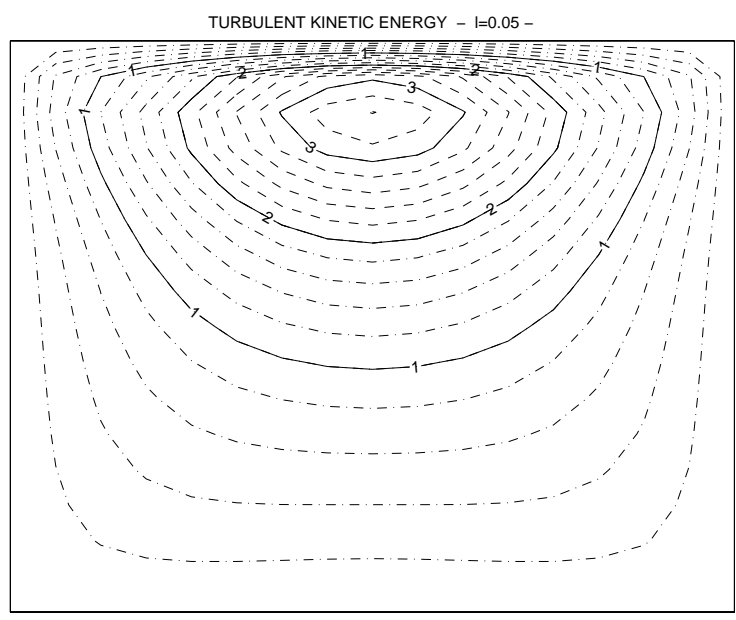

FIGURE 16.

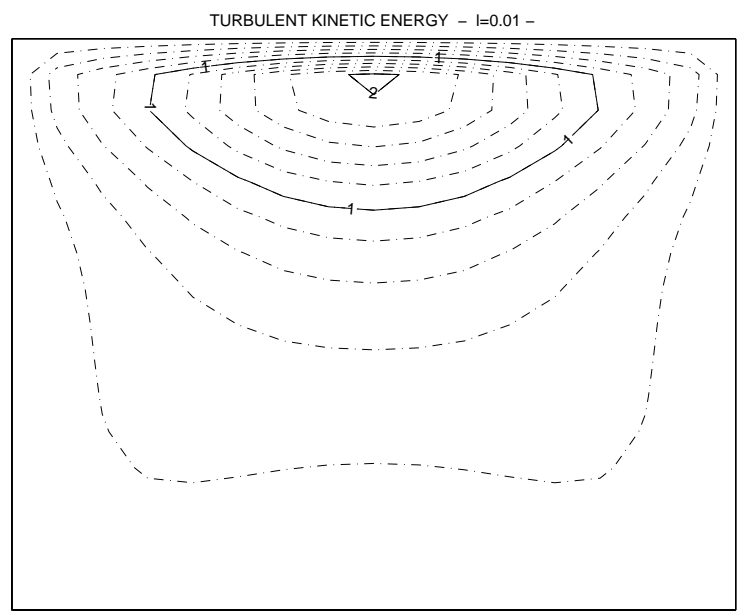

FIGURE 15.

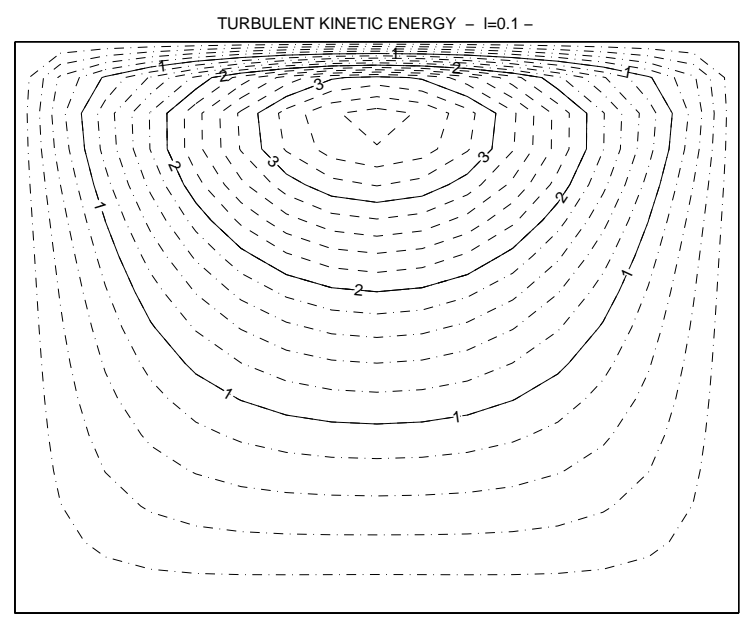

FIGURE 17.

of the solution even for large values of $\ell$ or at least uniqueness and regularity of the solution obtained by approximation. We recall that we have proved uniqueness only for $\ell$ sufficiently small and when the dissipation term $\varepsilon$ is neglected. When $\varepsilon$ is present, the equation governing kinetic energy $k$ degenerates as $\ell=0$. The turbulent model studied in this paper doesn't give rise to bifurcation branches, but regularizes the mean flow driven by a stationary forcing. Numerical experiments also point out the following convergence results: as $\ell \rightarrow \infty, \nu_{t}^{\ell} \rightarrow \infty, k_{\ell} \rightarrow 0$ and $u_{\ell} \rightarrow 0$. The convergence of $\left(k_{\ell}\right)$ is proved in Theorem 3.3, whereas for $\left(\nu_{t}^{\ell}\right)$ and $\left(u_{\ell}\right)$ we have proved only partial convergence results.

Acknowledgements. We warmly thank Daniel Martin for this help when using his numerical finite elements code MELINA. 


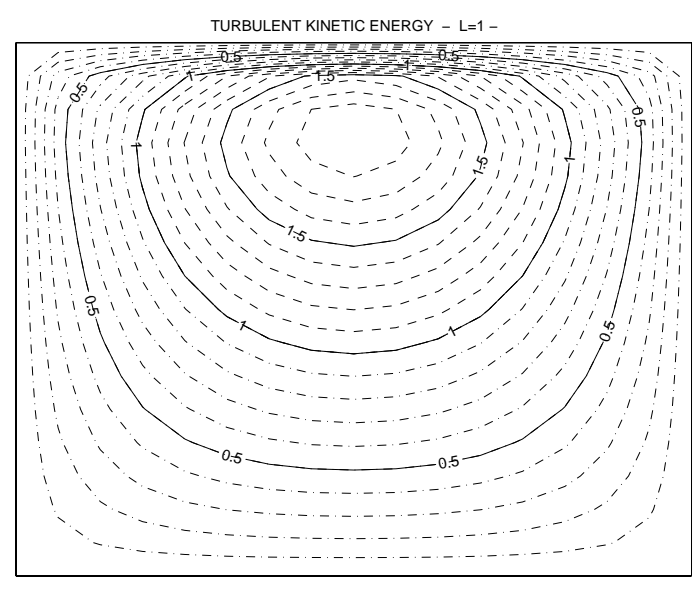

Figure 18.

TURBULENT VISCOSITY - $\mathrm{l}=0.1$ -

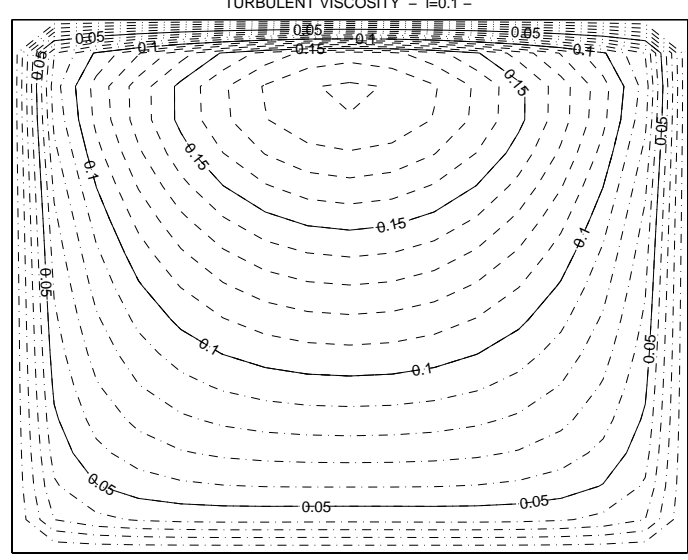

FiguRe 20.

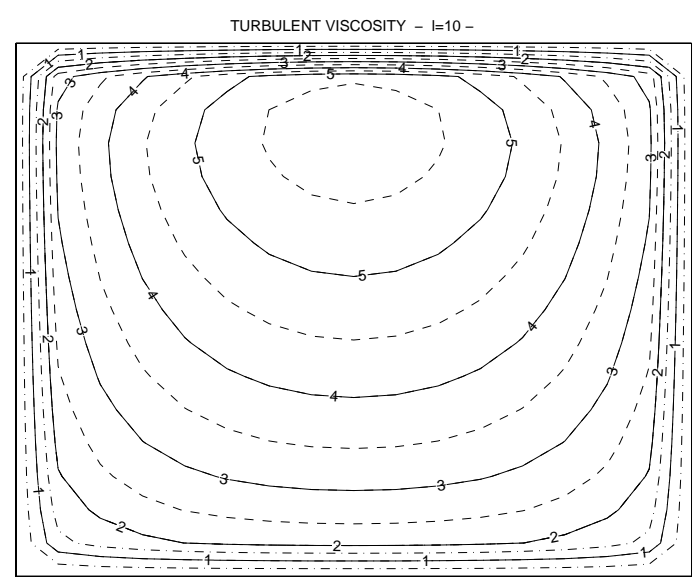

FIGURE 22.

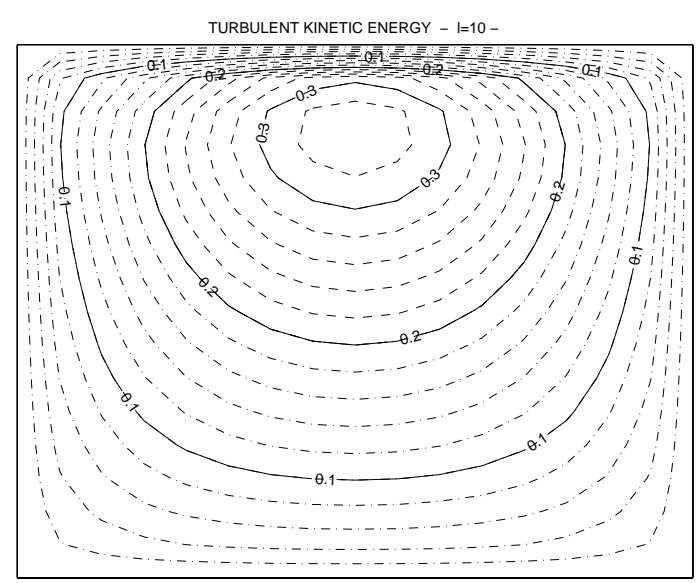

FIGURE 19.

TURBULENT VISCOSITY - l=1 -

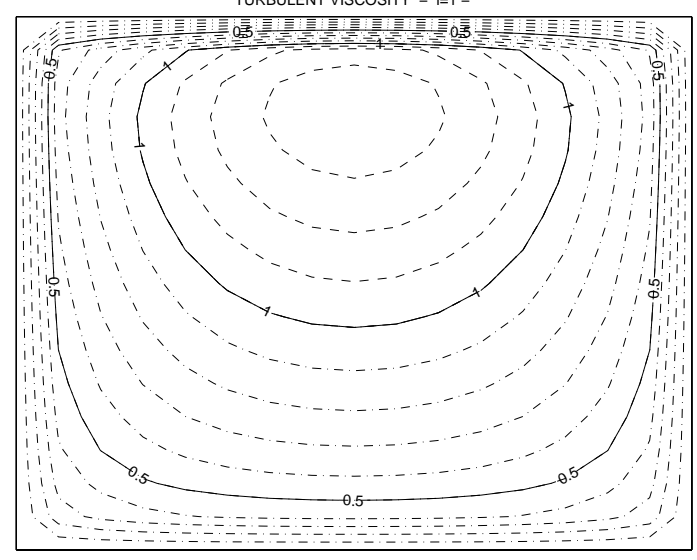

Figure 21.

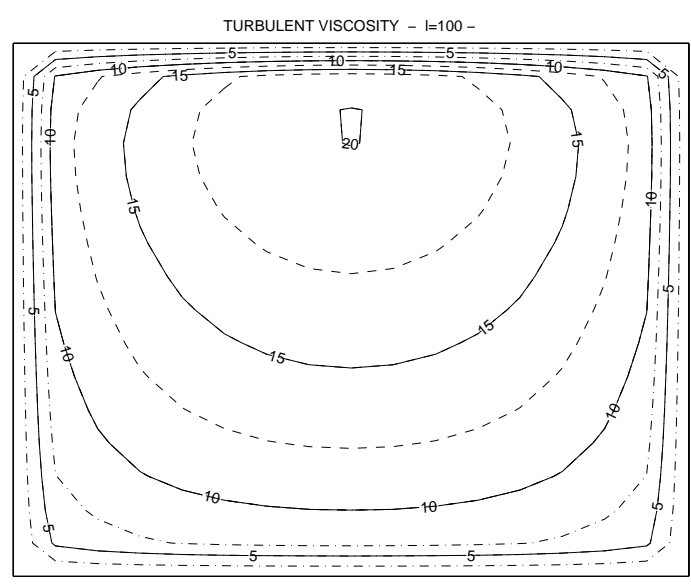

Figure 23. 


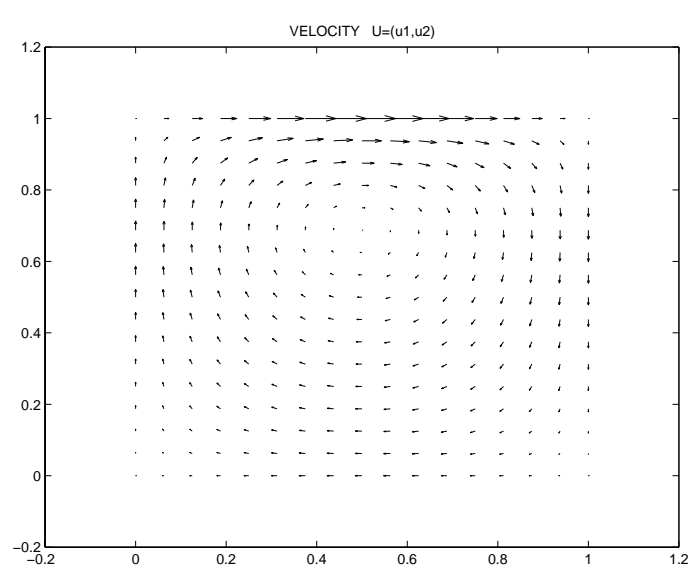

FigURE 24.

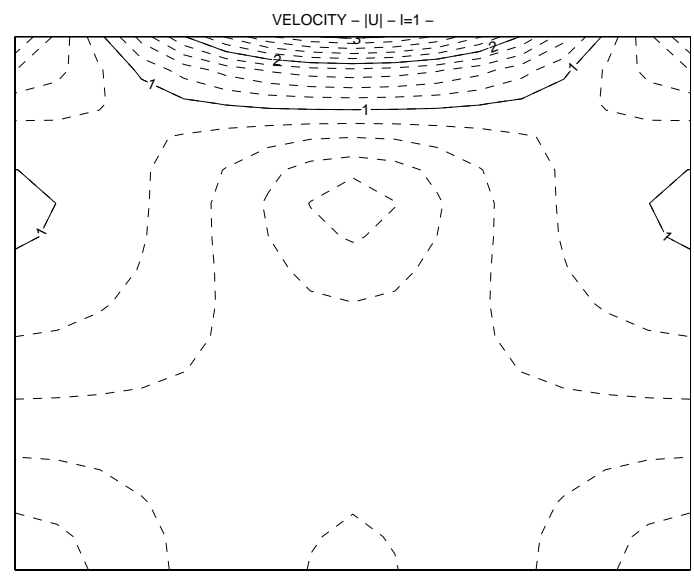

Figure 26.

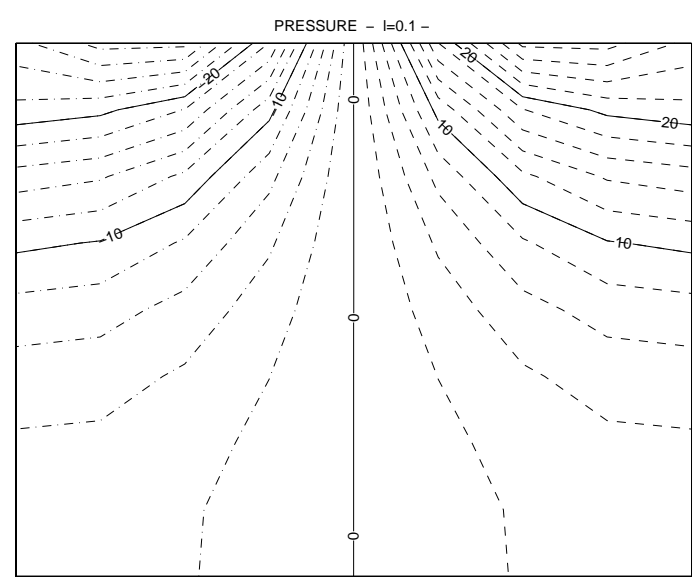

Figure 28.

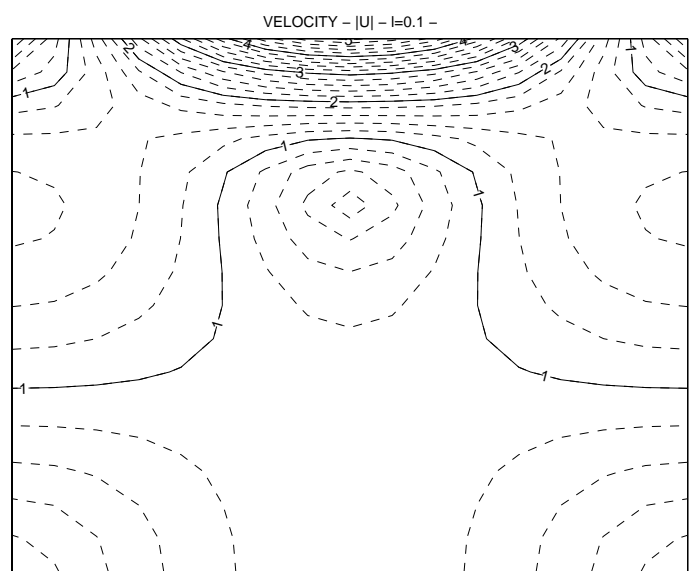

Figure 25 .

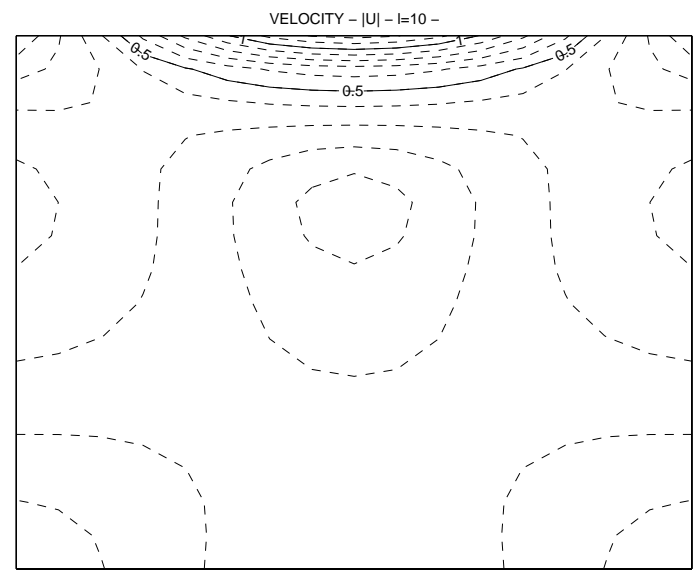

Figure 27.

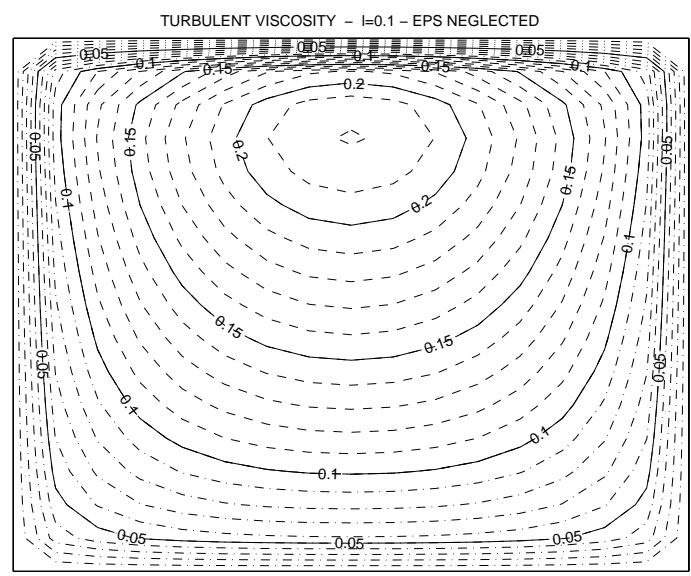

Figure 29. 


\section{REFERENCES}

[1] A. Belmiloudi and F. Brossier, Numerical study of a control method computing a three-dimensional flow from the observed surface pressure. IRMAR publication (2000).

[2] C. Bernardi, T. Chacon, F. Murat and R. Lewandowski, A model for two coupled turbulent fluids. Part II: Numerical analysis of a spectral discretization, to appear in Siam Journ. of Num. An. Part III: Numerical approximation by finite elements, submitted in Advance in Mathematical Science and Application.

[3] S. Clain and R. Touzani, Solution of a two-dimensional stationary induction heating problem without boundedness of the coefficients. RAIRO Modél. Math. Anal. Numér. 31 (1997) 845-870.

[4] J. Duchon and R. Robert, Inertial energy dissipation for weak solutions of incompressible Euler and Navier-Stokes equations. Nonlinearity 13 (2000) 249-255.

[5] T. Gallouët and R. Herbin, Existence of a solution to a coupled elliptic system. Appl. Math. Lett. 17 (1994) 49-55.

[6] T. Gallouët, J. Lederer, R. Lewandowski, F. Murat and L. Tartar, On a turbulent system with unbounded eddy viscosity, J. Nonlinear Analysis Theory, Methods and Analysis, in press.

[7] V. Girault and P.A. Raviart, Finite element methods for Navier-Stokes equations, theory and algorithm, Springer-Verlag (1986).

[8] R. Lewandowski, Analyse Mathématique et Océanographie. Collection RMA, Masson (1997).

[9] R. Lewandowski, The mathematical analysis of the coupling of a turbulent kinetic energy equation to the Navier-Stokes equation with an eddy viscosity. Nonlinear Anal. 28 (1997) 393-417.

[10] R. Lewandowski (in preparation).

[11] J.L. Lions and E. Magenes, Problèmes aux limites non homogènes et applications. Dunod (1968).

[12] D. Martin and Melina, http://www.maths.univ-rennes1.fr/ dmartin.

[13] B. Mohammadi and O. Pironneau, Analysis of the k-epsilon model. Collection RMA, Masson (1994).

[14] J. Oxtoby, Categories and measures. Springer-Verlag (1979).

[15] O. Pironneau, Méthode des éléments finis pour les fluides, Masson (1988).

To access this journal online: www.edpsciences.org 